\title{
Ire1 $\alpha$-Regulated Rate of mRNA Translation is Required for Acquisition of Identity and Polarity in Upper Layer Cortical Neurons
}

\author{
Mateusz C. Ambrozkiewicz ${ }^{1,, \$}$, Ekaterina Borisova ${ }^{1,2, *}$, Andrew G. Newman ${ }^{1}$, \\ Matthew L. Kraushar ${ }^{3,4}$, Theres Schaub ${ }^{1}$, Rike Dannenberg ${ }^{1}$, Marisa Brockmann ${ }^{5}$, \\ Marta Rosário ${ }^{1}$, Paul Turko ${ }^{6}$, Olaf Jahnn ${ }^{7,8}$, David R. Kaplan ${ }^{9}$, Takao Iwawaki ${ }^{10}$, \\ Christian M. T. Spahn ${ }^{4}$, Christian Rosenmund ${ }^{5}$, Victor Tarabykin ${ }^{1,2, \$}$
}

\footnotetext{
${ }^{1}$ Institute of Cell Biology and Neurobiology, Charité-Universitätsmedizin Berlin, corporate member of Freie Universität Berlin and Humboldt-Universität zu Berlin, Charitéplatz 1, 10117 Berlin, Germany,

${ }^{2}$ Institute of Neuroscience, Lobachevsky University of Nizhny Novgorod, pr. Gagarina 24, Nizhny Novgorod, Russian Federation,

${ }^{3}$ Department of Computational Molecular Biology, Max Planck Institute for Molecular Genetics, Ihnestraße 63-73, 14195 Berlin, Germany,

${ }^{4}$ Institute of Medical Physics and Biophysics, Charité-Universitätsmedizin Berlin, 10117 Berlin, Germany,

${ }^{5}$ Institute of Neurophysiology, Charité-Universitätsmedizin Berlin, 10117 Berlin, Germany,

${ }^{6}$ Institute of Integrative Neuroanatomy, Charité-Universitätsmedizin Berlin, 10117 Berlin, Germany,

${ }^{7}$ Proteomics Group, Max Planck Institute of Experimental Medicine, Hermann-Rein-Str. 3, 37075 Göttingen, Germany,

${ }^{8}$ Translational Neuroproteomics Group, Department of Psychiatry and Psychotherapy, University Medical Center Göttingen, Georg-August-University, von-Siebold-Str. 5, 37077 Göttingen, Germany

${ }^{9}$ Department of Molecular Genetics, University of Toronto, Canada,

${ }^{10}$ Medical Research Institute, Kanazawa Medical University, 1-1 Daigaku, Uchinada, Kahoku, Ishikawa 920-0293, Japan

*These authors contributed equally

${ }^{\$}$ Corresponding authors (email addresses: Mateusz C. Ambrozkiewicz, mateusz-

cyryl.ambrozkiewicz@charite.de; Victor Tarabykin, victor.tarabykin@charite.de)

Lead Contact: Victor Tarabykin
}

SUMMARY Establishment of cortical layers and axon-dendrite polarity in neurons is fundamental for brain connectivity. Here, we present that timed mRNA translation control by Inositol-Requiring Enzyme $1 \alpha$, Ire1 $\alpha$, is necessary for acquisition of upper layer neuronal identity and a single axon. We demonstrate that Ire $1 \alpha$ acts as a canonical regulator of global protein synthesis in developing cortical neurons, controlling the level of actively translating ribosomes, expression of translation factors, and ribosomal proteins. Translation rates distinguish early and late neuronal progenitors and early- and late-born postmitotic neurons, indicative of developmental stage- and differentiation-specific requirements for protein synthesis rates in the formation of upper and deeper cortical layers. We demonstrate that specification and polarization of upper layer neurons is uniquely sensitive to translation rate, in contrast to deep layer neurons. Our data shed light onto the post-transcriptional source of cellular diversity in the developing cortex and unveils stress-independent homeostatic functions of Ire1 $\alpha$. 


\section{Ire1 $\alpha$-Regulated Rate of mRNA Translation is Required for Acquisition of Identity and Polarity in Upper Layer Cortical Neurons}

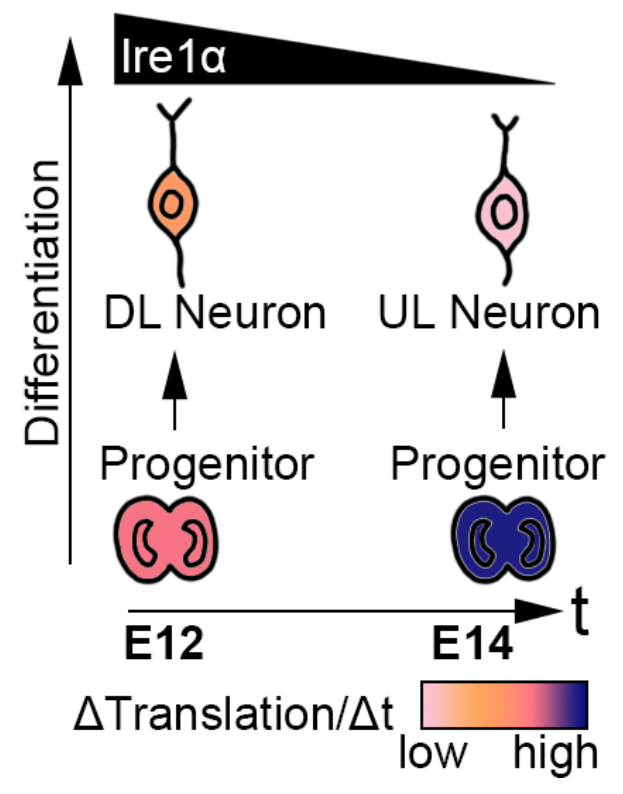

HIGHLIGHTS

- Small molecule screening reveals Ire $1 \alpha$ upstream of upper layer neuronal identity

- Axon acquisition in cortical neurons requires Ire1 $\alpha$

- Ire1 $\alpha$-regulated translation rate is a feature of early and late cortical progenitors

- Transient block of translation inhibits axon formation and upper layer identity

KEYWORDS: cortical development, neuronal fate, upper layers, polarity, axon, protein translation, neuronal progenitors, Ire1 $\alpha$, Satb2 


\section{INTRODUCTION}

The provenance of cellular diversity in the cerebral cortex has been the center of attention for developmental neuroscientists for decades. The mammalian neocortex, evolutionarily the newest add-on to the central nervous system, is organized in six laminae, each populated by

5 neurons with distinct afferents and efferent connectivity patterns, transcriptomic signatures, and electrophysiological properties. Pyramidal glutamatergic neurons of the neocortex are born from the progenitors at the ventricular side of the brain primordium and polarize synchronously with their migration to laminate distinct cortical layers. Deeper layers, forming subcortical tracts, are established first. Later born, upper layer neurons migrate through pre-

10 established deeper layers, and differentiate to extend polarized axons forming intracortical tracts, such as the corpus callosum (Arimura and Kaibuchi, 2005; Barnes et al., 2007; Götz et al., 1998; Malatesta et al., 2000; Rakic, 1972; Tarabykin et al., 2001).

Transcription factor signatures characterize pyramidal neurons of different layers. Special AT-Rich Sequence-Binding Protein 2 (Satb2) is one of the strongest determinant of

15 intracortically projecting pyramidal neurons identity and is required to from the corpus callosum (Alcamo et al., 2008; Britanova et al., 2008). Conversely, subcortical projections are determined by expression of FEZ Family Zinc Finger (Fezf) and COUP-TF-Interacting Protein 2 (CTIP2, also known as Bcl11b) in deep layer V and VI neurons, where loss of either gene disrupts the corticospinal tract (Arlotta et al., 2005; Chen et al., 2005; McKenna et al., 2015;

20 Molyneaux et al., 2005).

The temporal progression of ventricular progenitor state requires restriction of multipotency towards generating solely upper layer Satb2-positive neurons. Current models describe the temporal progression of neuronal differentiation as conserved across deep and upper layers, but the initial ground states embedded in their progenitors as progressively

25 discrete (La Manno et al., 2020; Nowakowski et al., 2017; Telley et al., 2019). Transcriptomic signatures in apical progenitors are temporally plastic, and able to adapt to the developmental milieu in elegant heterochronic transplantation experiments (Oberst et al., 2019). Transcriptional priming in progenitors involves translational repression which restricts expression of early lineage genes in the later born lineage (Zahr et al., 2018).

30 Intrinsic genetic programs along with extrinsic cues further establish axon-dendrite polarity and cortical neuron connectivity. Single axons guided through postmitotic differentiation programs specify their postsynaptic targets (Barnes and Polleux, 2009). Current challenges include determining how intra- and extracellular determinants of polarity interplay with the neuronal fate programs to establish neuronal networks during development.

35 The models of neuronal fate and connectivity specification summarized above have focused heavily on transcriptional measurements, leaving the post-transcription as an understudied layer of regulation in neocortical gene expression. We have previously demonstrated that RNA-binding proteins, translation, and ubiquitination pathways are major regulators of cortical connectivity, neuronal subtype specification, and axonal projections

40 (Ambrozkiewicz et al., 2018; Kraushar et al., 2014). In the mature brain, high-fidelity protein translation is proven essential to maintain synaptic transmission (Biever et al., 2020; Hafner et al., 2019). However, how translation regulates key developmental events, including neuronal polarization and the temporal succession of cortical progenitors, remains unclear. 
In this study, we sought upstream regulators of Satb2, which determines the

45 specification of callosally projecting cortical neurons (Alcamo et al., 2008; Britanova et al., 2008), and represents the late-stage restricted output of multipotent progenitors. Using a genetic reporter and Fluorescence Activated Cell Sorting (FACS), we screened a library of small molecules for their ability to alter Satb2 expression in developing neurons. Among our most impactful candidates was an inhibitor of kinase/RNase Inositol-Requiring Enzyme 1a

50 (Ire1a), also known as ER-to-Nucleus Signaling 1 (Ern1), the main sensor of ER lumen homeostasis and regulator of the Unfolded Protein Response (UPR). Upon ER stress, Ire1a signaling results in a translational shift promoting expression of proteins vital for cell survival and restoration of the ER folding capacity (Yoshida et al., 2001). Ire1a regulates stressindependent remodeling of actin filaments by its association with filamin A (Urra et al., 2018),

55 linked to periventricular heterotopia (Sheen, 2001). Another study unveiled a direct interaction of Ire1a with the ribosomes in vitro (Acosta-Alvear et al., 2018). Beyond that, homeostatic functions of Ire1a have remained quite elusive.

In this work, we demonstrate that Ire1 $\alpha$ is essential for acquisition of Satb2 neuronal identity during corticogenesis. In upper layer neurons, loss of Ire1 $\alpha$ is associated with loss of

60 axon polarity. Conditional deletion of Ire $1 \alpha$ in the developing neocortex changes the synthesis of ribosomal proteins and the levels of actively translating ribosomes. Using fluorescent noncanonical amino acid tagging (FUNCAT), we show that Ire1a modulates translation rates in cortical progenitors and neurons. Moreover, we reveal that the rate of protein synthesis distinguishes early and late neuronal progenitors and their derived postmitotic neurons.

65 Transient inhibition of translation early during development results in loss of Satb2 expression and axon polarity. Taken together, our study defines translational signatures of neuronal progenitors and distinct neuronal subtypes, unveiling the layers of gene expression regulation driving neuronal diversity during corticogenesis. Additionally, this study extends the function of Ire1 1 beyond stress pathways to an innate developmental signal for neuronal specification

70 in the cortex.

\section{RESULTS}

\section{Ire1 $\alpha$ is a positive regulator of Satb2 and axon specification in developing cortical neurons}

75 In order to identify signaling pathways orchestrating neuronal subtype specification, we have previously developed a method for screening small molecule inhibitors for their role in the control of cell fate acquisition (Ambrozkiewicz et al., 2017), which utilizes the Satb2 ${ }^{\text {Cre/+ }}$ mouse line (Britanova et al., 2006). By expressing a Cre-inducible fluorescent reporter loxPStop-loxP-tdTomato in Satb2 ${ }^{\mathrm{Cre} /+}$ primary cortical cells (Fig. 1a), we used cell sorting to

80 quantify the proportion of Satb2-expressing tdTomato-positive cells (Satb2 ${ }^{\text {tdTom}}$ ) to EGFPexpressing cells. We used EGFP-encoding reference plasmid to normalize for the transfection efficiency.

We prepared Satb2 ${ }^{\mathrm{Cre} /+}$ primary cortical cells from E13.5 embryos and screened a library of 450 kinase inhibitors for their ability to alter the proportion of Satb2 ${ }^{\text {tdTom }}$ neurons at 85 day-in-vitro two (DIV2) (Grinshtein et al., 2016). APY69 was among the strongest modulators of Satb2 expression with its dose-dependent effect (Fig. 1b, Table S1). APY69 specifically 
inhibits Ire $1 \alpha$, an evolutionarily ancient bimodal transmembrane kinase and RNase, which acts as an endoplasmic reticulum (ER) stress sensor, where it catalyzes mRNA splicing in a spliceosome-independent manner (Lee et al., 2008; Tirasophon et al., 1998). Recent

90 evidence highlights an unfolded protein response (UPR)-independent developmental function of Ire $1 \alpha$ as regulator of cellular migration (Urra et al., 2018). We hypothesized that stress-independent function of Ire $1 \alpha$ might be essential for specification of Satb2 identity in developing neurons.

We first studied the expression pattern of Ire1 $\alpha$. Fluorescence in situ hybridization

95 (FISH) revealed a homogenous expression of Ire1 $\alpha$ throughout the developing cortex. We noted a strong developmental downregulation of Ire $1 \alpha$ in the cortex, with its highest levels at E12.5, both at the level of protein, and mRNA (Fig. 1c-1d). As opposed to isolated perinuclear puncta visible in the majority of cells, we observed single cells at E12.5 and at E14.5 with diffuse cytoplasmic Ire1 $\alpha$ mRNA FISH signal.

100 We then went on to validate the pharmacological screening results for APY69 (Fig. 1b) using a knock-out (KO) mouse model. Given ubiquitous expression of Ire1 $\alpha$ in many tissue types, its full $\mathrm{KO}$ results in overt developmental defects, associated with non-central nervous system-related phenotypes (Iwawaki et al., 2009). To carve out the role of Ire $1 \alpha$ in cortical development, we used the Ire $1 \alpha^{f / f}$ line, which enables Cre-dependent deletion of

105 exons 20-21 from the floxed Ire $1 \alpha$ allele. Upon expression of Cre, the kinase-extension nuclease (KEN) domain including RNase active site is deleted from the Ire1 $\alpha$ protein.

To inactivate Ire $1 \alpha$ in defined cell populations in the developing cortex, we took advantage of in utero electroporation (IUE), which allows for in vivo DNA delivery to a spatiotemporally defined subset of progenitors at the ventricular surface of the embryonic

110 cortex. Early (E12.5) neuronal progenitors display multipotency in their ability to generate both CTIP2- and Satb2-positive neuronal progeny, whereas late (E14.5) progenitors give rise to exclusively Satb2-expressing neurons populating upper layers. In our first set of experiments, we used IUE in Ire $1 \alpha^{\mathrm{f} / \mathrm{f}}$ mice to transfect EGFP as a control and both EGFP and Cre to induce Ire $1 \alpha \mathrm{KO}$ in a subset of early cortical progenitors at E12.5 and derived progeny

115 (Fig. 1e). We then immunostained E16.5 coronal cortical sections for EGFP and neuronal identity markers, Satb2 and CTIP2. We first noted disrupted, highly branched morphology of Ire $1 \alpha \mathrm{KO}$ neurons, compared to majority of bipolar control cells (Fig. 1f). Quantification revealed fewer Satb2-expressing neurons at the expense of CTIP2-positive ones in the Ire1 $\alpha$ $\mathrm{KO}$ (Fig. 1g), concordant with our in vitro results (Fig. 1b). On the contrary, overexpression

120 of human IRE1 $\alpha$ in wild-type early cortical progenitors at E12.5 (Fig. 1h) resulted in an increased proportion of Satb2-expressing neurons at E18.5, and remarkably, hardly any CTIP2-positive neurons (Fig. 1i).

Post-mitotic promoter NeuroD1-induced expression of Cre in Ire $1 \alpha^{\mathrm{f} / \mathrm{f}}$ at E12.5 did not alter the proportion of Satb2 and CTIP2 neurons at E16.5 (Fig. S1a and S1b), indicative of a

125 neuronal progenitor-embedded role of Ire1 $\alpha$ in the acquisition of neuronal identity. We observed an analogous morphological phenotype in NeuroD1-Cre expressing neurons in Ire $1 \alpha^{\mathrm{f} / \mathrm{f}}$ mice as in ones transfected with CAG-Cre (Fig. S1a and S1c, compare with Fig. 1f). 
Using an Emx1-Cre deleter, we then conditionally inactivated Ire1 $\alpha$ in the progenitors of dorsal telencephalon by establishing a Ire $1 \alpha^{\mathrm{f} / \mathrm{f} ;} ; \mathrm{Em} \times 1^{\mathrm{Cre} /+}$ mouse line, Ire $1 \alpha \mathrm{cKO}$. We

130 injected pregnant mice with BrdU at E12.5 (Fig. S2a-S2d) and E14.5 (Fig. S2e-S2h) and quantified the proportions of Satb2 and CTIP2-expressing neurons at P2, when neurogenesis is complete. Among all E12.5-derived BrdU+ cells, we counted fewer Satb2+ and more CTIP2+ cells (Fig. S2b and S2c), consistent with our IUE experiments (Fig. 1). Strikingly, in E14.5 BrdU-pulsed P2 embryos, we found slightly more BrdU+/CTIP2+ cells (Fig. S2f and $135 \mathrm{~S} 2 \mathrm{~g}$ ), which localized to the top of the CP.

These data altogether indicate a critical requirement of Ire1 $\alpha$ for the correct proportion between upper layer-associated Satb2-positive and deeper layer-associated CTIP2expressing neurons specified during cortical development. 
a Small molecule screening in Satb2 ${ }^{\mathrm{Cre} /+}$

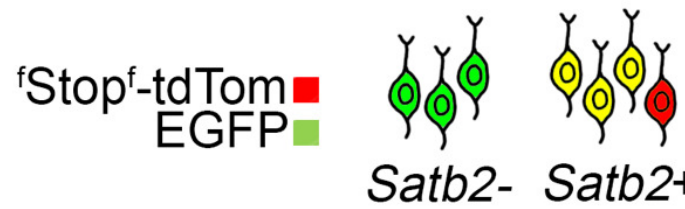

b DMSO

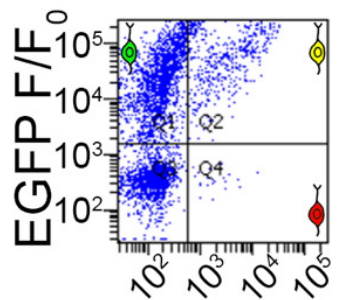

FACS Satb2 ${ }^{\mathrm{t} T \mathrm{~T}} \mathrm{~m}$
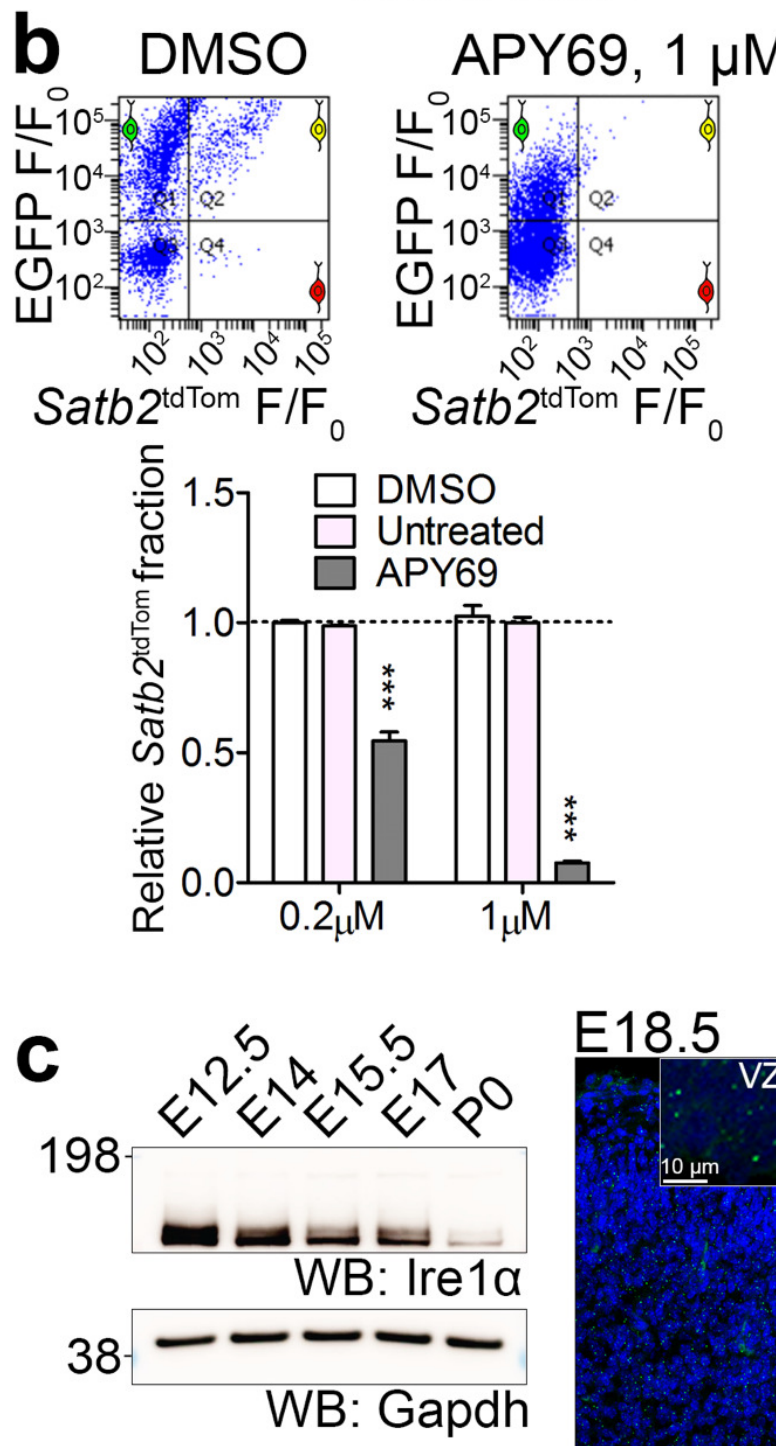

E14.5

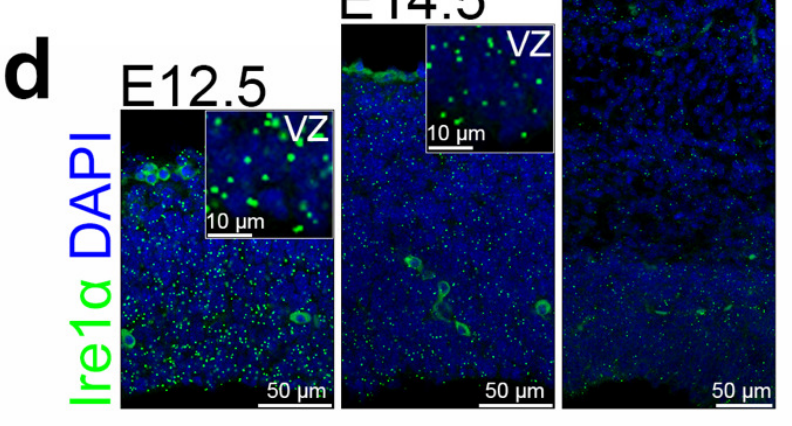

e IUE E12.5 - E16.5
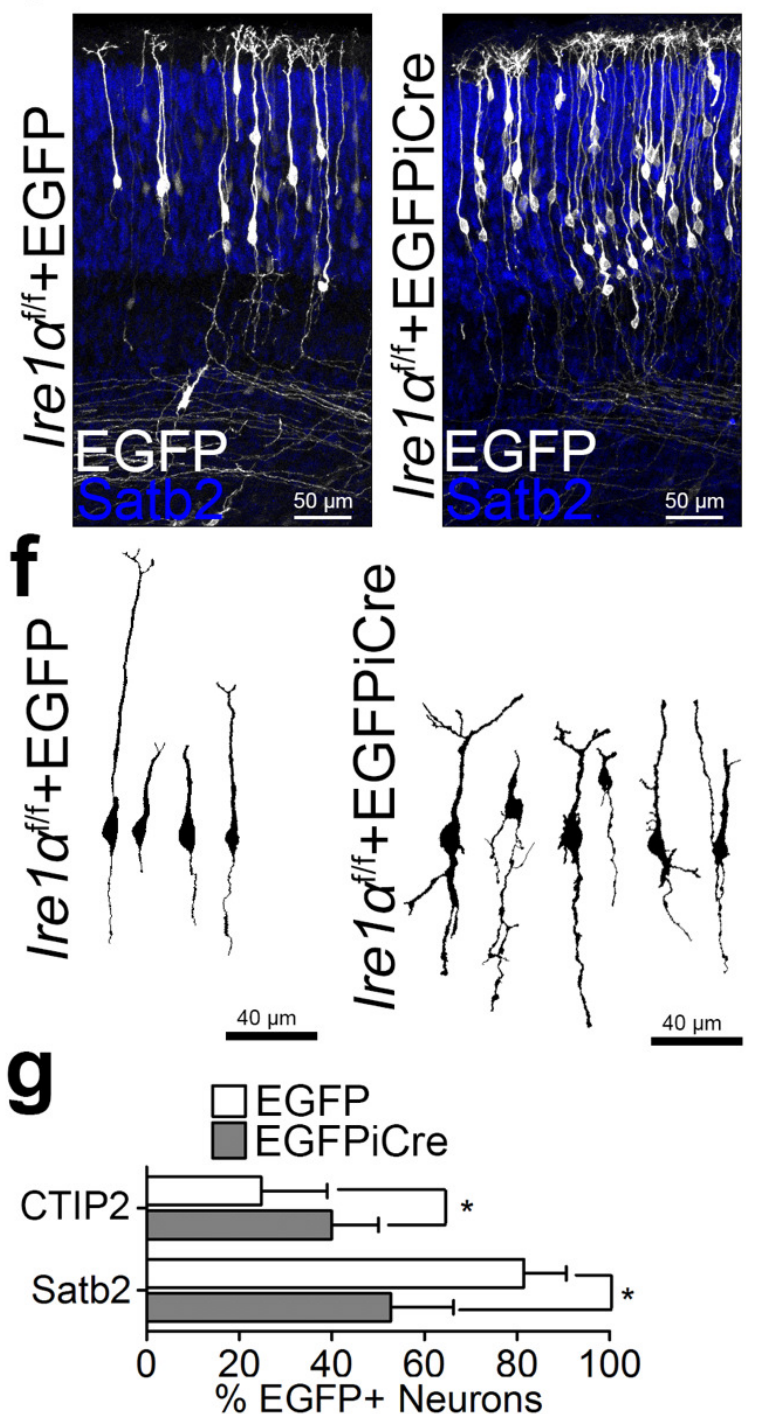

h

IUE E12.5 - E18.5
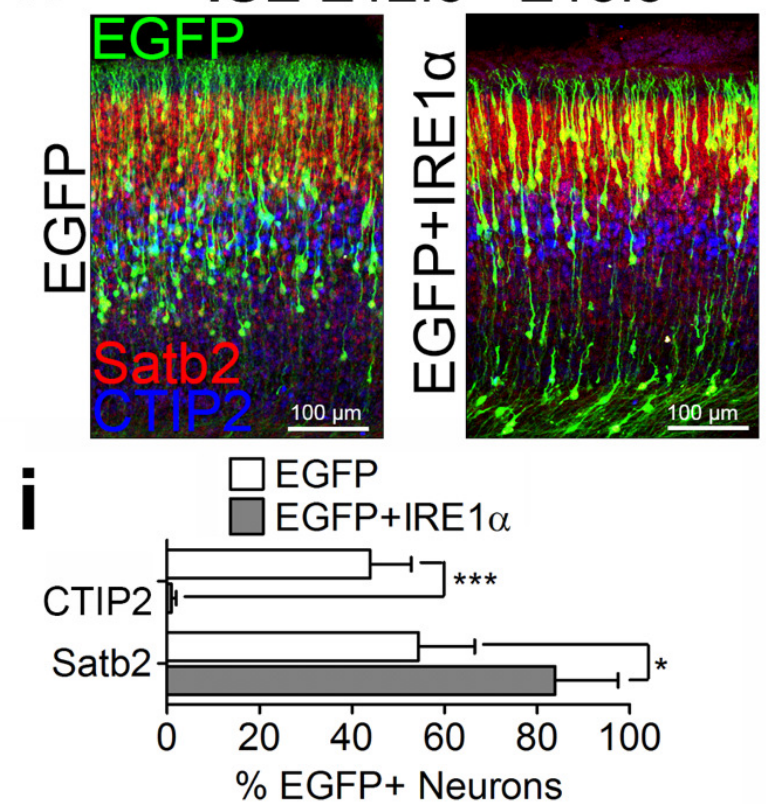
Fig. 1. Small molecule screening reveals Ire1 $\alpha$ activity pivotal for upper layer neuronal identity and bipolar morphology of cortical neurons.

(a) Screening workflow to identify signaling pathways upstream of Satb2 neuronal identity. (b) Top 145 panels: representative results of flow cytometry of DIV2 neurons treated with DMSO and APY69, a selective inhibitor of Ire1 $\alpha$. Bottom panels: quantification of the proportion of Satb2-positive neurons. (c) Western blotting in cortical lysates to profile developmental expression of Ire1 $\alpha$. Gapdh, loading control. (d) Fluorescence in situ hybridization for Ire1 $\alpha$ in cortical sections at indicated developmental stages. Insets demonstrate enlarged fragments of the ventricular zone (VZ). (e) Representative

150 images of immunostaining against EGFP and Satb2 in E16.5 coronal cortical sections of Ire $1 \alpha^{\mathrm{f} / \mathrm{f}}$ embryos after IUE at E12.5 with plasmids encoding for EGFP or EGFP and Cre simultaneously. (f) Representative EGFP-based tracings of single neurons after IUE in (e). (g) Quantification of neuronal cell identity after IUE described in (e). (h) Representative images of cortical coronal sections at E18.5 after IUE in wild-type E12.5 embryos to express EGFP, or EGFP and human IRE1 $\alpha$. (i) Quantification

155 of neuronal cell identity after IUE described in (h). For (e), (g) and (h-i), compare Fig. S6. Bar graphs show averages \pm S.D. Statistics for (b), one-way ANOVA with Bonferroni post-hoc test; $(\mathrm{g})$ and (i), D'Agostino-Pearson normality test and unpaired t-test. ${ }^{* * *} p<0.001 ; 0.01<{ }^{*} p<0.05$. 

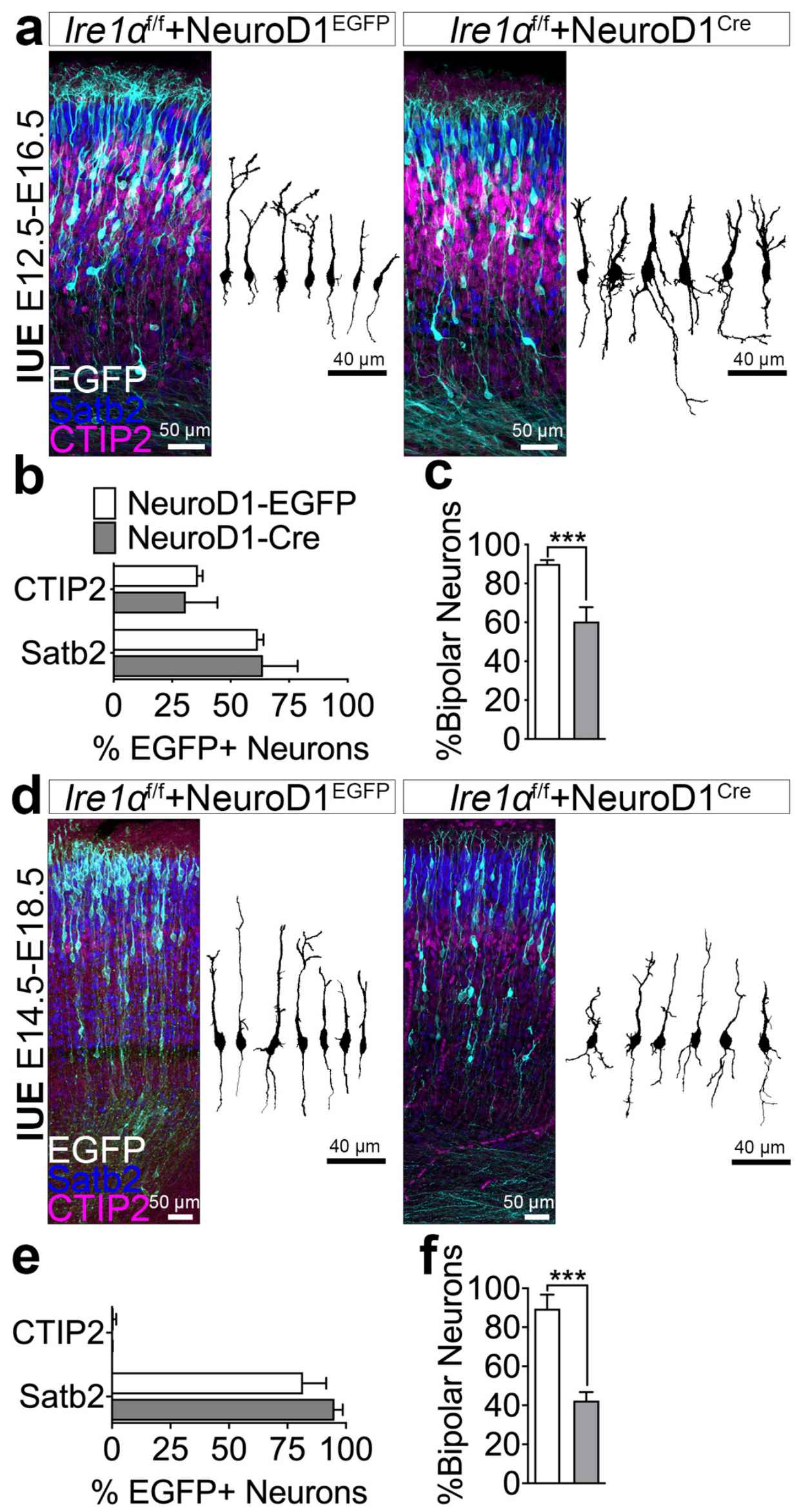
Figure S1. Related to Fig. 1. Ire1 $\alpha$-driven acquisition of the upper layer identity, but not of bipolar morphology, is embedded in neuronal progenitors.

(a, d) Representative images of immunostaining in coronal cortical sections from E16.5 (a) or E18.5 (d) Ire1 $\alpha^{\text {f/f }}$ embryos after IUE at E12.5 (a) or E14.5 (d) with plasmids encoding for EGFP under the 165 promoter of NeuroD1 gene or Cre under the promoter of NeuroD1 together with loxP-Stop-loxPEGFP. Shown are also representative, EGFP-based tracings of single neurons. (b, e) Quantification of neuronal cell identity in (a) and (d). (c, f) Average proportion of bipolar neurons in (a) and (d). Bar graphs show averages \pm S.D. For statistical analyses, D’Agostino-Pearson normality test and MannWhitney test. ${ }^{* *} p<0.001$.

170 

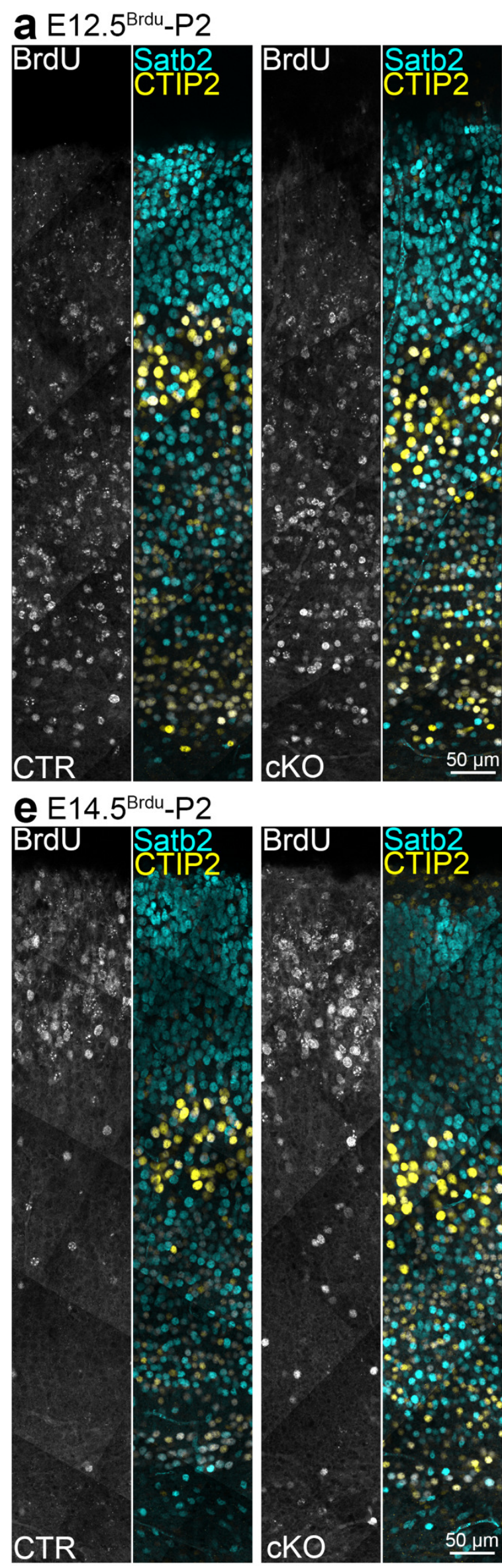
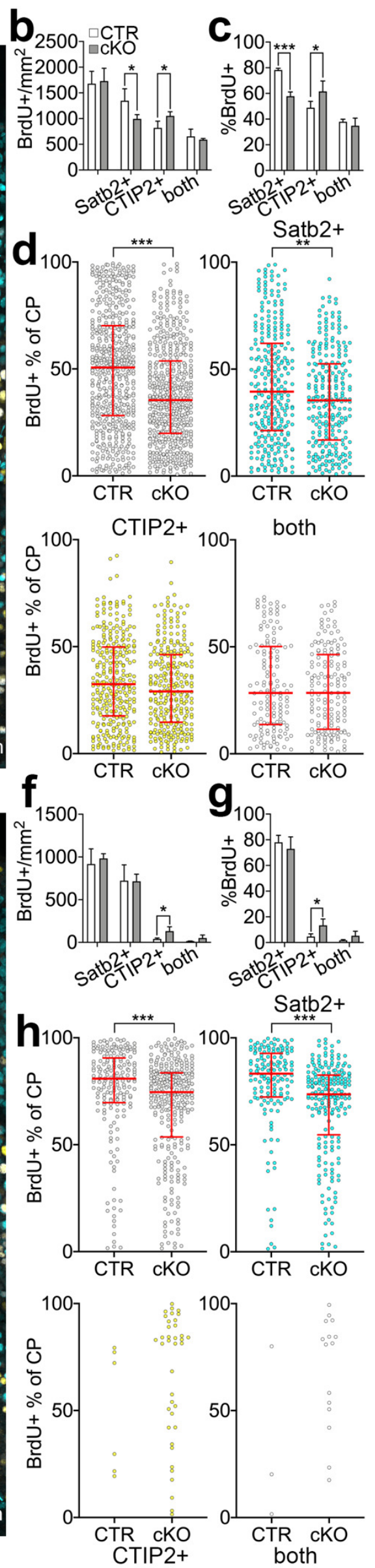
Figure S2. Related to Fig. 1. Birth dates and laminar positions of Satb2+ and CTIP2+ neurons in Ire1 $\alpha$ cKO cortex.

(a, e) Representative images of immunostaining in control or cKO coronal cortical sections after BrdU 175 injection at E12.5 (a-d) or E14.5 (e-h). (b), (f) Quantification of cell density expressing an indicated nuclear stain. "Both", cells co-expressing of Satb2 and CTIP2. (c), (g) Quantification of the proportion of neurons expressing indicated marker within all BrdU+ cells. (d), (h) Laminar distribution of neurons expressing indicated marker. The position of each neuron was normalized to the thickness of the CP and represented as a dot (100\% CP - pia, 0\% CP - bottom of the CP). Graph contains pooled data

180 from indicated number of brains (Table S1). Bar graphs show averages \pm S.D. Line and error bars on (d) and (h) indicate median and interquartile range. For statistical analyses, D'Agostino-Pearson normality test and (d) and (h), Mann-Whitney test; (b-c) and (f-g) unpaired t-test. $0.001<{ }^{* *} p<0.01$; ${ }^{* * *} p<0.001$. 


\section{Ire $1 \alpha$ is required for axon specification in developing cortical neurons of upper layers}

Efferent cortical connectivity is established by the correct proportion of neuronal types and specification of functional axons during development. Because of the prominent morphological phenotype for E12.5 progenitor-derived neurons (Fig. 1f), and given that

190 pyramidal upper layer neurons serve as an established model to study specification of axondendrite polarity (Barnes and Polleux, 2009), we electroporated E14.5 neuronal progenitors of Ire $1 \alpha^{\mathrm{f} / \mathrm{f}}$ embryos with plasmids encoding EGFP or EGFP and Cre and fixed the brains at E18.5. In line with previously published results (Urra et al., 2018), loss of Ire1 $\alpha$ lead to a disrupted laminar positioning of upper layer cortical neurons. While control neurons primarily

195 localized to the top of the cortical plate (CP), as predicted from their birthdate at E14.5, we noted a substantial number of Cre-expressing neurons populating the bottom of the CP as well (Fig. 2a and 2b). Consistently, in the cKO brains, both E12.5 and E14.5 BrdU-pulsed Satb2-expressing cells localized to the lower portions of the CP, as compared to the control cells (Fig. S2). Using expansion microscopy (ExM), we detected multiple trailing processes

200 (TPs) and many short processes originating from the soma in Ire $1 \alpha \mathrm{KO}$ upper layer neurons (Fig. 2c). This contrasted with the majority of control neurons characterized with a single TP and lack of additional neurites emanating from the soma at this stage (Fig. 2d). Disruption of bipolar morphology and aberrant laminar positioning within the CP was independent of Satb2 expression (Fig. 2e). We also observed morphological defects induced by IUE of NeuroD1-

205 Cre inducing Ire $1 \alpha \mathrm{KO}$ in differentiating neurons (Fig. S1d-S1f). These data indicate Ire1 $\alpha$ has both a progenitor and post-mitotic neuron embedded role in the regulation of neuronal morphology during cortex development.

To study the identity of excessive processes specified by Ire $1 \alpha \mathrm{KO}$ neurons, we took advantage of ex utero electroporation (EUE), which permits modulation of gene expression

210 in the neuronal progenitors followed by in vitro primary neuronal culture. We expressed EGFP or simultaneously EGFP and Cre in E14.5 upper layer neuron progenitors of Ire $1 \alpha^{\mathrm{f} / \mathrm{f}}$ embryonic cortices and cultured isolated primary cells until DIV4, when axons are established in control neurons (Ambrozkiewicz et al., 2018). As expected, the majority of control cells projected a single longest neurite, while Ire $1 \alpha \mathrm{KO}$ cells were symmetrical and failed to project

215 a long axon (Fig. 2f). We then immunostained the fixed cell cultures for axonal and dendritic markers (Tau-1 and MAP2, respectively) and counted the number of axons projected by single neurons (Fig. $2 \mathrm{~g}$ ). We failed to identify an axon in a fraction of Ire $1 \alpha \mathrm{KO}$ neurons, and identified a significant proportion of neurons that projected multiple axons, as compared to polarized control cells (Fig. 2h). Where present, Ire1 $\alpha \mathrm{KO}$ axons were shorter (Fig. 2i) and

220 more branched (Fig. 2j), as compared to control ones. Ire $1 \alpha \mathrm{KO}$ neurons with no axons exhibited increased dendritic branching, possibly implicating an axon-to-dendrite identity switch of single neurites. Axon possessing Ire $1 \alpha \mathrm{KO}$ neurons exhibited a normal dendritic tree, when compared to controls (Fig. $2 \mathrm{k}$ and 2l). This indicates formation of excess axons upon Ire $1 \alpha \mathrm{KO}$, rather than a dendrite-to-axon transition in neurons with multiple axons.

225 Morphological defects in the KO were associated with somatic distribution of ER tubules visualized by EGFP-Sec61 $\beta$ expression as compared to its polarized localization in neurites 
of control cells (Fig. $2 \mathrm{~m}$ and 2n). This indicates a possible involvement of Ire $1 \alpha$ in ER-driven neuronal polarization.

We then asked if such profound morphological defects have consequences on axon-

230 related neurophysiology. At DIV6, axons of upper layer control neurons are enriched for Tau1 (Fig. S3a), Ankyrin G (Fig. S3b), and voltage-gated $\mathrm{Na}^{+}$channels ( $\mathrm{Na}_{\mathrm{v}}$ s; Fig. S3c). In Ire $1 \alpha$ $\mathrm{KO}$ neurons, we observed a perinuclear somatic localization of these axonal markers, indicative of their aberrant subcellular localization. Current clamp recordings from Ire $1 \alpha^{\text {f/f }}$ autaptic hippocampal neurons infected with lentiviruses encoding for EGFP or Cre showed a

235 higher number of action potentials (APs) generated upon lower current injections (Fig. S3d and S3e). This might be attributable to more axon initial segments (AIS) specified by Ire $1 \alpha$ $\mathrm{KO}$ neurons and/or their altered molecular composition, which increases the chance of firing at lower currents. Importantly, overall membrane integrity and conductance of Ire $1 \alpha \mathrm{KO}$ neurons were unaltered (Fig. S3f and S3g). Altogether this evidence reveals that loss of

240 bipolar morphology in developing Ire $1 \alpha \mathrm{KO}$ neurons is associated with defects in axon specification, subcellular mislocalization of axonal markers and increased current sensitivity. 

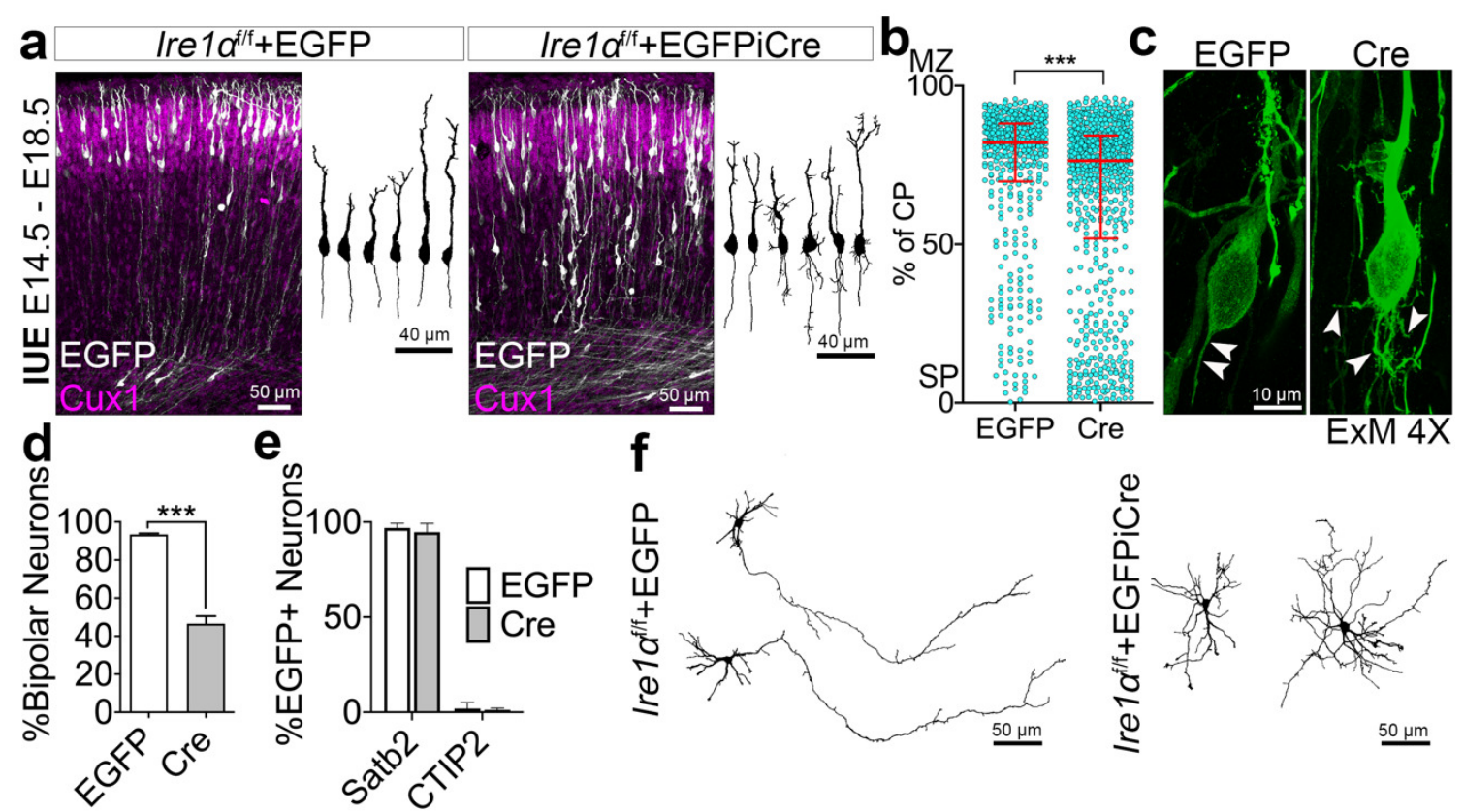

g/re $1 a^{\text {f/f }}+$ EGFP Single axon
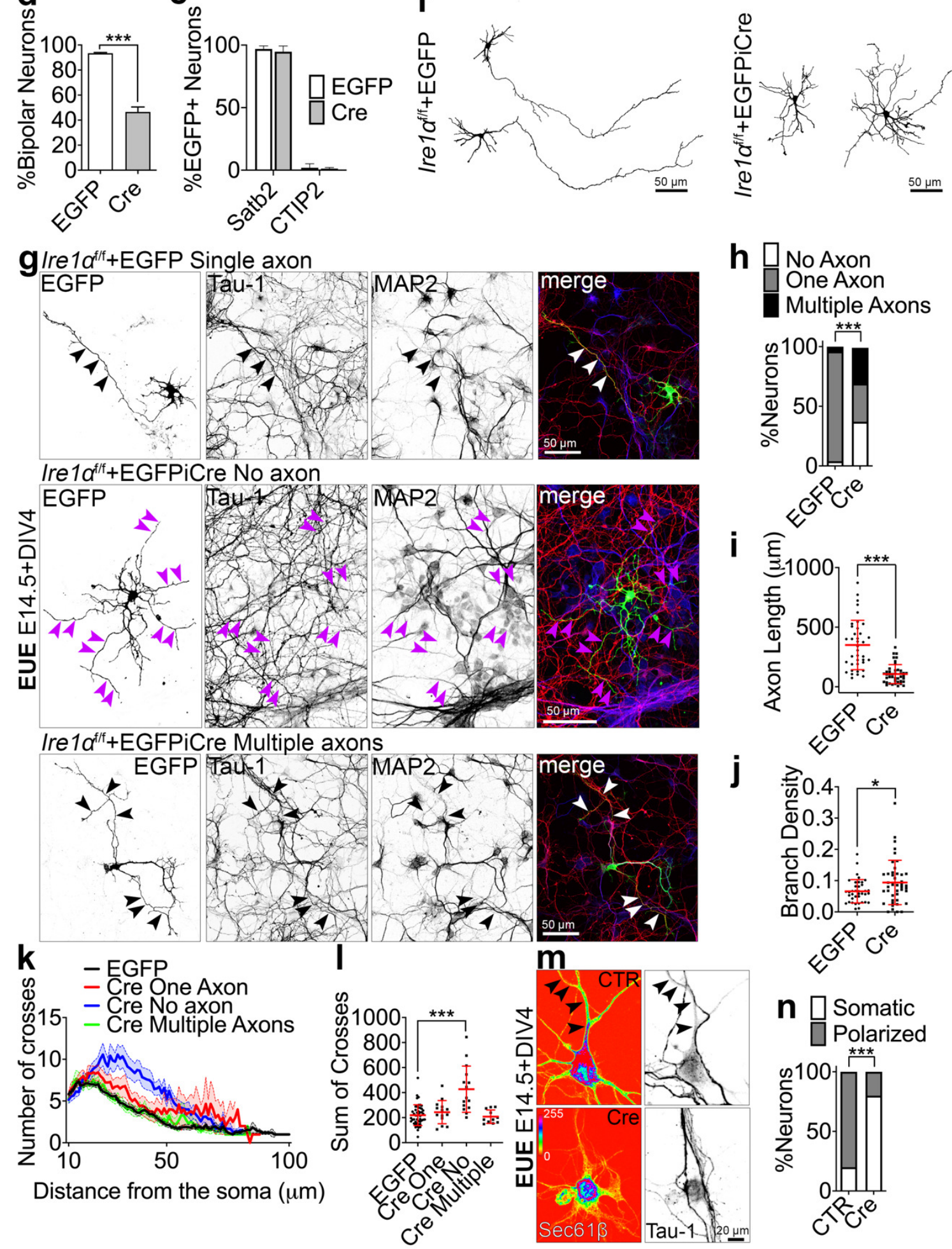
h $\square$ No Axon One Axon
Multiple Axons

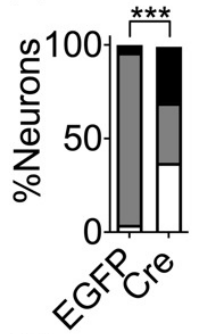

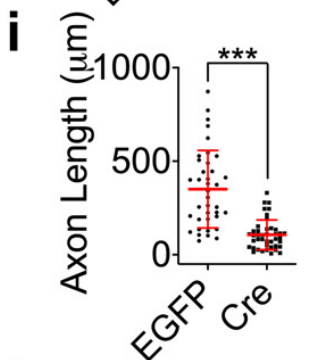
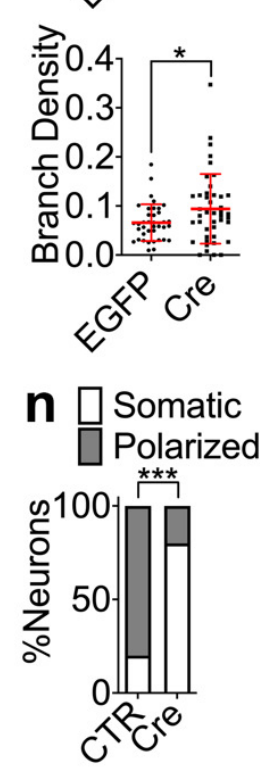
Fig. 2. Ire $1 \alpha$ is indispensable for bipolar morphology, axon specification and proper laminar 245 positioning of upper layer neurons.

(a) Representative images of EGFP and upper layer-expressed Cux1 immunostaining in coronal cortical sections from e18.5 Ire $1 \alpha^{\text {f/f }}$ embryos after IUE at e14.5 with indicated plasmids and semiautomatic, EGFP-fluorescence based tracings of single neurons. (b) Laminar distribution of cortical neurons in e18.5 Ire $1 \alpha^{\mathrm{f} / \mathrm{f}}$ brains after IUE described in (a). The position of each neuron was normalized 250 to the thickness of the CP and represented as a dot. 0\% - Subplate (SP), 100\% - Marginal Zone (MZ). Graph contains pooled data from indicated number of brains (Table S1). (c) Representative images of EGFP immunostaining after expansion microscopy (ExM) in cortical sections from (a). Arrowheads point to trailing processes. (d) Average proportion of bipolar neurons from (a). (e) Quantification of neuronal identities in experiment in (a). (f) Representative EGFP-based tracings of $I r e 1 \alpha^{\mathrm{f} / \mathrm{f}}$ neurons at 255 DIV4 after EUE to express EGFP or EGFP and Cre. (g) Neurons in (f) were fixed and immunostained with axonal or dendritic markers. White and black arrowheads indicate axons, purple ones indicate neurites lacking Tau-1 expression. (h) The number of axons projected from a single neuron. Average axon length (i), average axon branch density per micrometer (j), Sholl analysis diagram (k) and average sum of crossing dendrites (I) in neurons expressing EGFP or EGFP and Cre, projecting no, 260 one, or multiple axons. (m) Representative EGFP signals and Tau-1 immunostaining of Ire $1 \alpha^{\mathrm{f} / \mathrm{f}}$ DIV4 neurons after EUE to co-express EGFP-Sec61 $\beta$ and empty vector (CTR) or Cre. ( $n$ ) Quantification of EGFP-Sec61 $\beta$ localization in experiment in $(m)$. Line and error bars on (b) indicate median and interquartile range, scatter plots in (i), (j), (l) and bar graphs on (d) and (e) show averages \pm S.D. Results on (k) are represented as averages \pm S.E.M. Statistics for (b), (d-e), (i-j), D'Agostino-Pearson 265 normality test and Mann-Whitney or unpaired t-test; (h), Chi-square test; (I), Kruskal-Wallis with Dunn's multiple comparisons test; $(n)$, Fisher's test. ${ }^{* * *} p<0.001 ; 0.01<{ }^{*} p<0.05$. 


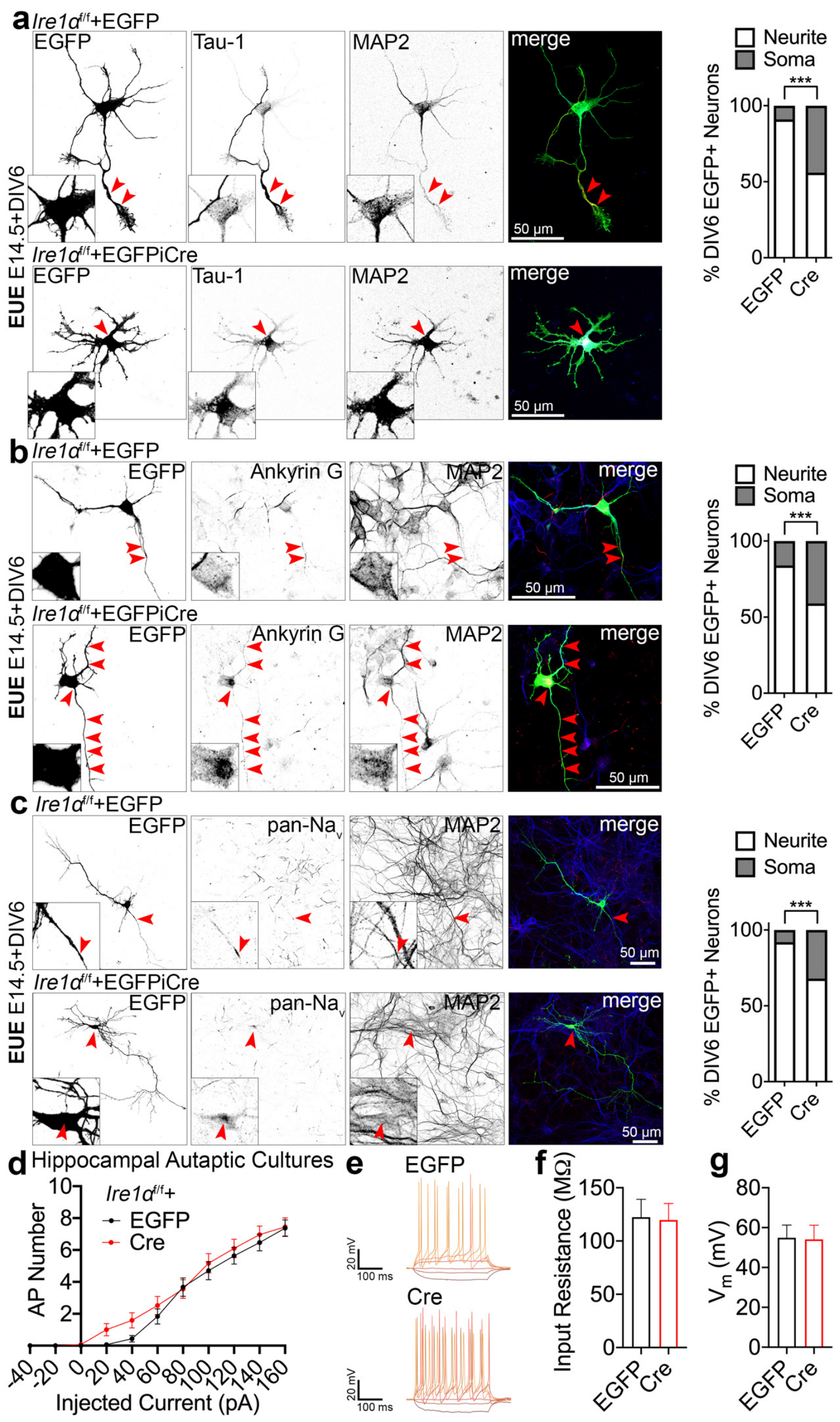


Fig. S3. Related to Fig. 2. Loss of Ire1 $\alpha$ disrupts axon initial segment and current responses. 270 (a-c) Images of representative DIV6 primary cortical Ire $1 a^{f / f}$ neurons after EUE at E14.5 for EGFP or EGFP and Cre expression. Neuronal cultures were immunolabeled for indicated axonal and dendritic marker proteins. Graphs show quantification of the subcellular distribution of axonal markers. Ire $1 \alpha-$ deficient neurons display somatic accumulations of Tau-1, Ankyrin $\mathrm{G}$ and $\mathrm{Na}_{\mathrm{v}}$. Red arrowheads indicate enrichment of axonal markers. Insets on the bottom left of black and white images are zoom-

275 ins to the somata or places of axonal marker accumulation. (d-g) Electrophysiological recordings from autaptic hippocampal cultures prepared from P0 Ire $1 \alpha^{\mathrm{f} / \mathrm{f}}$ pups. Neuronal cultures were infected with lentiviruses encoding for EGFP or Cre. (d) Quantification of the number of APs generated after injection of increasing amount of current. (e) Example traces of recordings described in (d). Average input resistance ( $f$ ) and holding potential $(g)$. Data points and error bars on (d) represent mean \pm 280 S.E.M. and graphs on (f-g), mean \pm S.D. For statistical analyses, (a-c), Fisher's test; (d), one-way ANOVA; (f-g), Mann-Whitney test. ${ }^{* * *} p<0.001$. 


\section{Ire1a drives upper layer specification and axon formation in the neocortex by controlling expression levels of translation regulators}

285 During the UPR, robust cellular reprogramming is driven by Ire1 $\alpha$ signaling network (Cox et al., 1993; Mori et al., 1993). Ire1 $\alpha$ activation is paralleled by suppression of general translation (Di Prisco et al., 2014). We hypothesized that also during development, Ire1a orchestrates upper layer subtype and axonal polarization by influencing protein translation and thereby affecting expression of key developmental determinants. To test this, we analyzed the

290 ribosome profile in forebrain-specific Ire1a cKO, and we purified actively translating ribosomes ex vivo (Kraushar et al., 2021). Given a scarcity of material at early embryonic stages, we used E18.5 brains. Sucrose density gradients revealed higher level of polysomes in Ire1a cKO cortices (Fig. 3a).

We then investigated which RNAs localize to polysome fraction in Ire1 $\alpha$ cKO cortex.

295 We performed RNA sequencing in bulk cortex of control and cKO as well as in the polysome fraction purified from the control and Ire1 $\alpha$ cKO cortices. Notably, we did not detect gross changes in the level of the transcriptome in the bulk RNAseq [(Fig. 3b; cutoff at corrected pvalue 0.05, AY036118 IncRNA outlier comes from inflated expression counts given its alignment with Rn45s repeat (Kimmel et al., 2019)]. Polysome-associated RNA in Ire1 $\alpha$ cKO

300 cortices (depicted as colored data points, cutoff at corrected p-value 0.05 ) encoded for structural components of the ribosome, proteins acting on RNA, revealed by the gene set enrichment analysis (GSEA, Fig. 3d), and other molecules regulating translation and initiation factors (Table S2). These results indicate that Ire1a loss in the cortex alters translation of RNAs crucial for ribosome complex function.

305 We then investigated the expression levels of canonical translation regulators using Western blotting and E18.5 cortical lysates (Fig. S4a and S4b). We detected no gross changes in the level of classical regulators of translation in the developing cortex, like S6-P, Fragile X Mental Retardation Protein 1 (FMRP), or eukaryotic initiation factor $2 \alpha$. Notably, the level of spliced Xbp1, a cellular stress-associated Ire1a substrate was not altered, indicating

310 a non-canonical UPR-independent role of Ire1a in the regulation of polysome level. We identified upregulated levels of eukaryotic elongation factor 2 (eEF-2), downregulated phosphorylated eEF2 on Thr56 and decreased level of eukaryotic initiation factor 4A1 (elF4A1) in the Ire1 $\alpha$ cKO cortex (Fig. 3e and 3f). Given no gross changes on the RNA level (Fig. 3b), we conclude that the expression level changes for these factors are of translational

315 nature.

To mimic the cKO scenario, we then overexpressed eEF-2 using IUE in e12.5 and e14.5 wild type embryos (Fig. S4c-S4g). Four days later, we detected no alteration of Satb2 proportion in the neuronal progeny after eEF-2 OE (Fig. S4d and S4f). However, we did detect a positioning defect of upper layer neurons, similar to the one observed upon loss of Ire1a

320 (Fig. S4g). Next, we inactivated elF4A1 using CRISPR-Cas9 technology (Fig. 3g), which allows for simultaneous sgRNA and humanized Cas9 nickase delivery to developing cortex using IUE in e12.5 wild type embryos (Shinmyo et al., 2016). The loss of elF4A1 resembled Ire1 $\alpha \mathrm{KO}$ (Fig. 1e and 1g) regarding the types of neurons generated, with less Satb2expressing neurons, at the expense of CTIP2-positive cells (Fig. 3h). Loss of elF4A1 from

325 upper layer progenitors did not affect laminar positioning in the cortex at e18.5 (Fig. S4eS4g). Both elF4A1 and eEF-2 were highly and homogenously expressed in the E12.5 cortex, 
and downregulated at E14.5, resembling the expression pattern of Ire1a (Fig. S4h-S4i, compare Fig. 1d). We also studied the polarity acquisition in DIV4 neurons after EUE-induced eEF-2 OE, eIF4A1 KO and simultaneous eEF-2 OE and elF4A1 KO (Fig. S4j-S4k). We found

330 that all three manipulations lead to emergence of neurons with 0 axons, characterized by absence of Tau-1 enrichment in a single neurite, partially reminiscent of Ire $1 \alpha \mathrm{KO}$ neurons (Fig. 2g-2h). Notably, simultaneous eEF-2 OE and elF4A1 KO resulted in the highest proportion of neurons without an axon.

Altogether, these findings demonstrate that Ire1a controls expression levels of 335 translation regulators. In particular, initiation factor elF4A1 acts downstream of Ire1 $\alpha$ to control Satb2 expression and thereby neuronal subtype identity determination in the developing neocortex, while elongation factor eEF-2 regulates the laminar distribution of upper layer neurons. Tightly regulated levels of both elF4A1 and eEF-2 in neuronal progenitors are critical for specification of an axon in developing neurons. 


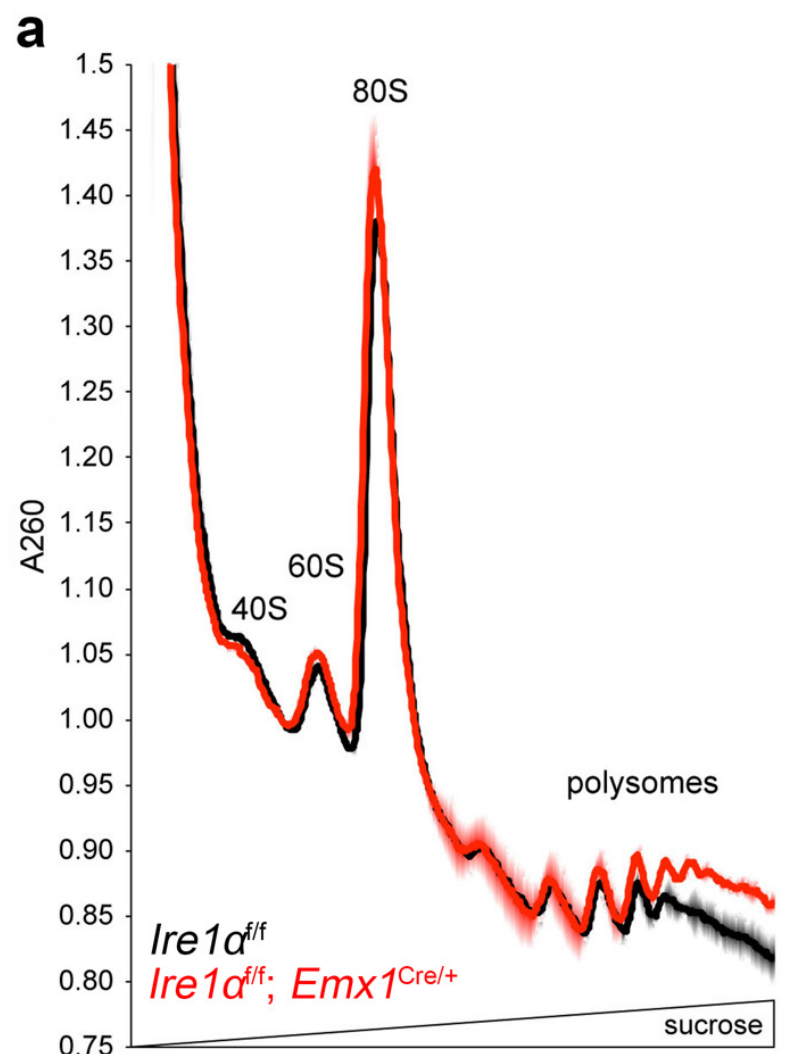

d GO Cat. Activity GO Structural C. Acting on RNA of Ribosome
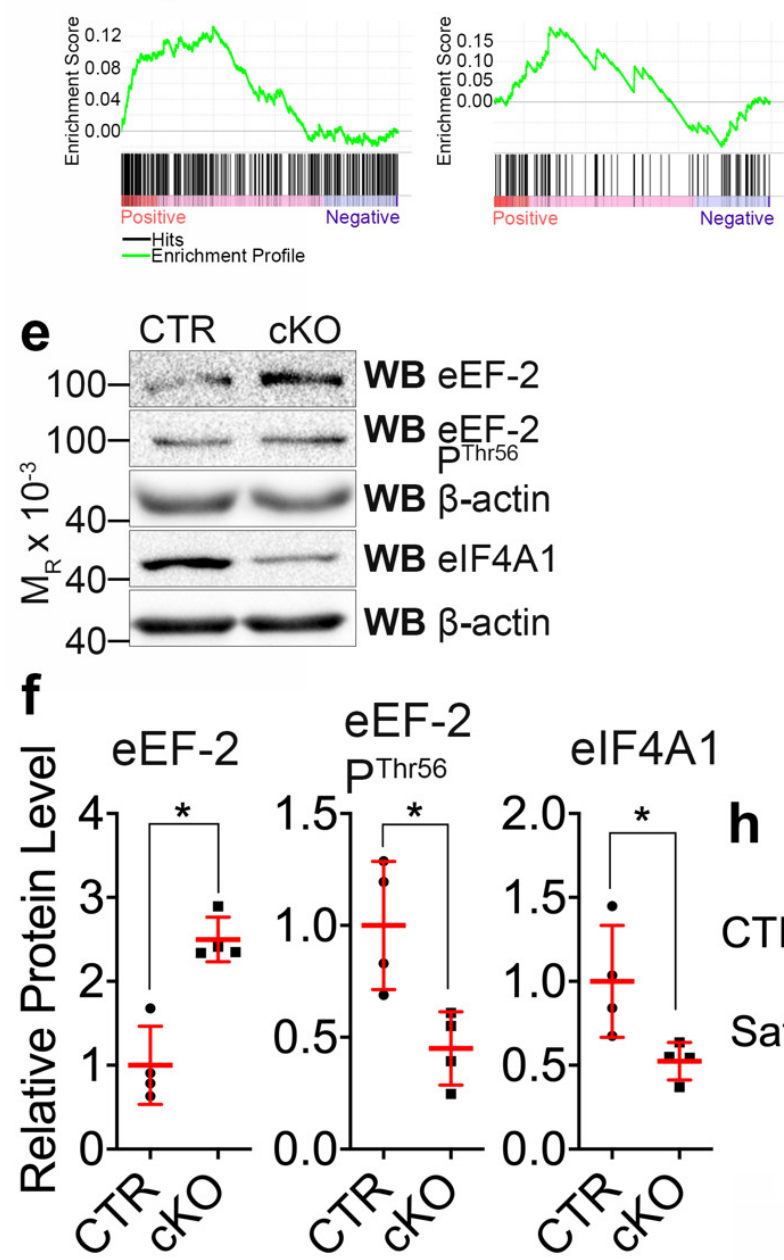

b $_{10.0}$ Bulk RNAseq

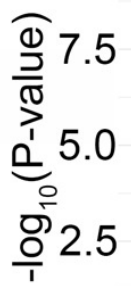

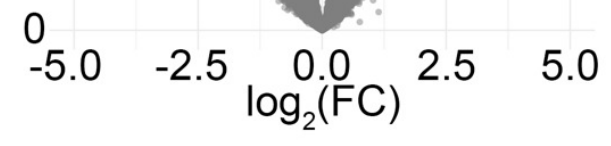

C Polysome RNAseq


IUE E12.5 - E18.5

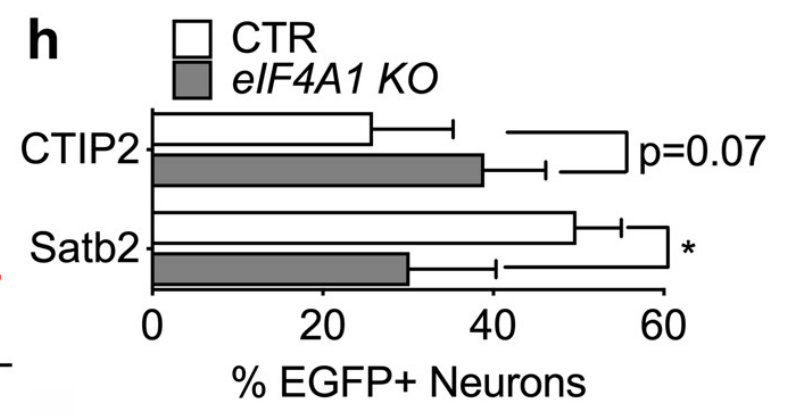


Fig. 3. Ire1 $\alpha$-mediated protein translation regulation drives upper layer identity acquisition in the cortex.

(a) Analytic density gradient fractionation of A260-normalized E18.5 neocortex lysates, measuring the 345 relative abundance of ribosomal subunits, $80 \mathrm{~S}$ ribosomes, and polysomes. A260 curves plotted as mean \pm S.D. across replicate fractionations $(n=2$, each comprising 7-8 brains per condition), baseline (1.0) centered at onset of $40 S$ peak. ( $b$ and c) Volcano plots for Ire1 $\alpha$ cKO RNAseq in bulk tissue (b) and polysome-enriched RNA (c). Colored data points represent significantly regulated genes. FC; fold change. All P-values are Benjamini-Hochburg adjusted p-values. (d) Top two GSEA enrichment plots

350 for cKO polysome RNA fraction versus control polysome RNA fraction, Catalytic Activity Acting on RNA and Structural Component of Ribosome (Table S2). (e) Representative Western blotting results using the control and Ire1 $\alpha$ cKO E18.5 cortical lysates. (f) Quantifications of the protein level from (e). (g) Representative images of immunostaining against EGFP and CTIP2 in E18.5 coronal cortical sections of wild type embryos after IUE at E12.5 with gRNAs and Cas9 nickase to achieve indicated 355 genotypes. (h) Quantification of neuronal cell identity in the experiment in (g). Compare Fig. S6. Red line and error bars on (f) and bar graphs of $(h)$ indicate mean \pm S.D. Statistics for $(f)$ and $(h)$, D'Agostino-Pearson normality test and Mann-Whitney test. $0.01<{ }^{*} p<0.05$. 

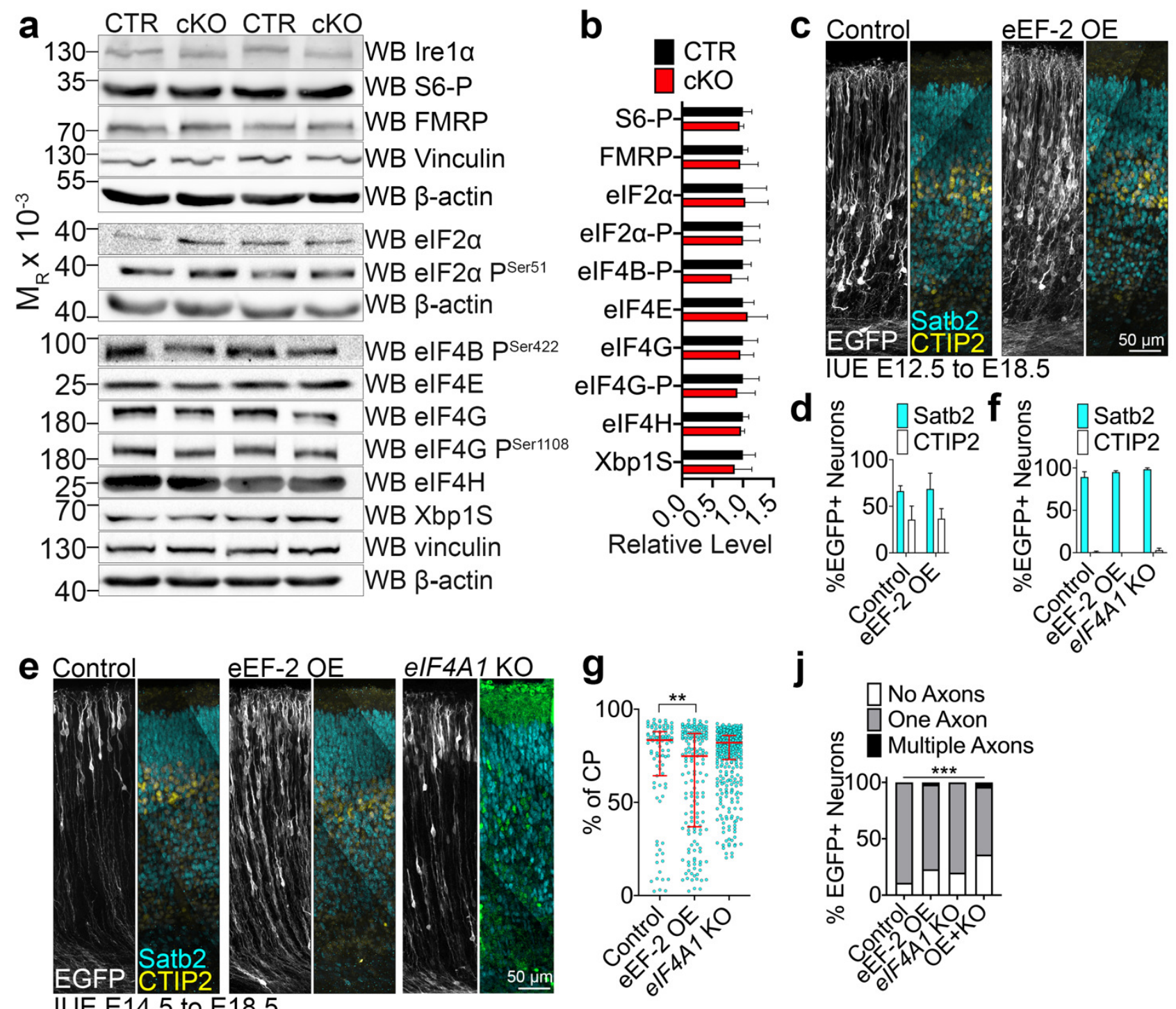

j
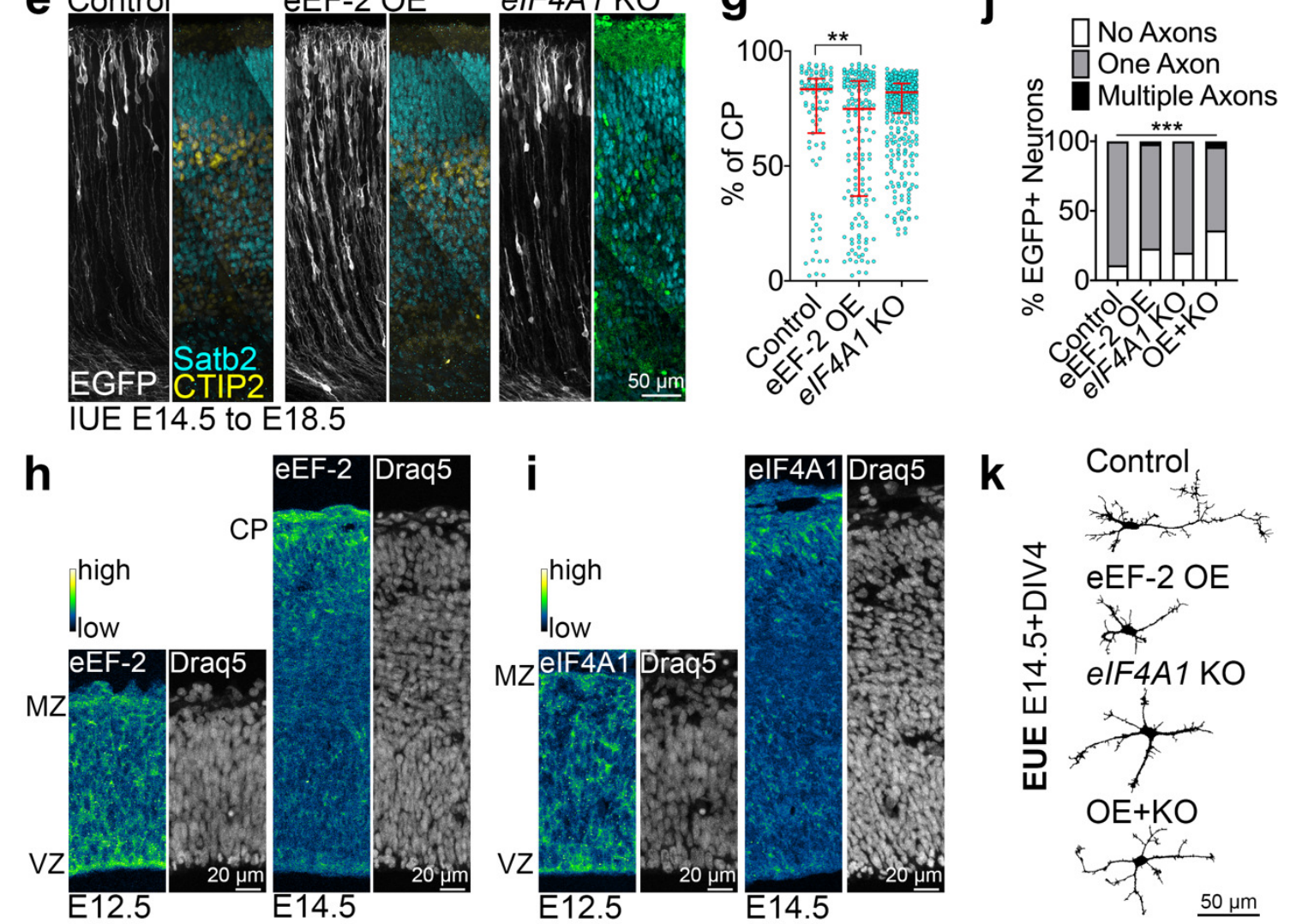

Fig. S4. Related to Fig. 3. Ire1a-mediated protein translation regulation employs stressindependent signaling pathways and drives axon specification.

(a) Representative results of Western blotting in E18.5 cortical lysates from control and Ire1a cKO using indicated antibodies. (b) The expression level of analyzed protein was normalized to the amount 365 of beta-actin in the sample and expressed as relative protein level. (c-g) Representative images (stitched tiles) of EGFP fluorescence signals and immunolabeling for Satb2 and CTIP in E18.5 brains 
of wild type embryos after IUE at E12.5 (c-d), or E14.5 (e-g) with plasmids encoding for EGFP (Control) or for EGFP and eEF-2 (eEF-2 OE), or for EGFP and elF4A1 gRNAs and Cas9 nickase (elF4A1 KO). Quantification of neuronal identity ( $d$ and $f$ ) and positioning within the cortical plate $(\mathrm{g})$. 370 (h-i) Representative images of the immunostaining in the coronal cortical slice for indicated proteins and Draq5, a nuclear marker. VZ, ventricular zone; MZ, marginal zone; CP, cortical plate. Graph contains pooled data from indicated number of brains (Table S1). (j) The number of axons projected from a single neuron at DIV4 after EUE to achieve indicated genotypes. Neurons were fixed and immunolabeled from axonal and dendritic markers (compare with Fig. 2). OE+KO, simultaneous eEF-

3752 OE and elF4A1 KO. (k) Representative EGFP-based tracings of DIV4 neurons from (j). Apart from Control, shown are neurons with 0 axons. Bar graphs indicate mean \pm S.D. Line and error bars on $(\mathrm{g})$ indicate median and interquartile range and scatter plots individual positions of single neurons. For statistics on (b) unpaired t-test with post-hoc Holm-Sidak for multiple comparisons; (d), Mann-Whitney test; (f), (g), Kruskal-Wallis test with Dunn's multiple comparisons; (j), Chi-square test. $0.001<{ }^{* *} p<$ $3800.01 ;{ }^{* * *} \mathrm{p}<0.001$. 


\section{Ire1 $\alpha$ is a regulator of protein translation rates in the developing cortex}

Increased polysome level in the Ire $1 \alpha$ cKO may represent ribosomes elongating more slowly or stalling, and thus accumulating in the heavy fraction (Eshraghi et al., 2021). To test this

385 hypothesis, we examined translation rates in Ire1 $\alpha$ cKO neurons using FUNCAT (Beatty and Tirrell, 2008; Dieterich et al., 2010). The method relies on replacing L-methionine with its alkyne analog L-homopropargylglycine (HPG) in the medium of primary cultured cells, and HPG detection in a Huisgen alkyne-azide cycloaddition reaction. Within a cell surface area, the fluorescence intensity of azide-coupled Alexa-647 is proportional to the amount of

390 incorporated HPG into newly synthesized proteins in defined time and serves as an estimate of translation rate in a given cell (Fig. 4).

We focused our analyses on a uniform cell population to circumvent a possibility of confounding effects of varying translation rates in different cell types. Control of neuronal identity mediated by Ire1a is embedded in early neuronal progenitors (Fig. 1g and S1b). For

395 this reason, we first investigated protein synthesis rates using EUE to induce Ire1a loss in early E12.5 multipotent neuronal progenitors. At DIV1, we quantified lower translation rates in Cre-expressing mitotic marker Ki67-positive (Scholzen et al., 2002) Ire1a KO progenitors as compared to EGFP-expressing Ire1 $\alpha^{\mathrm{f} / \mathrm{f}}$ ones (Fig. 4a). Similarly, we detected lower translation rates in E12.5 mitotic progenitors upon loss of elF4A1 (Fig. 4c and 4d). We

400 quantified approximately $50 \%$ lower rates of translation in DIV4 upper layer Ire $1 \alpha$ cKO neurons born from E14.5 cortical progenitors as compared to control cells (Fig. 4e and 4f). Taken together, regardless of the cell type, Ire1 $\alpha$ loss engenders a decrease in the rate of protein synthesis. In the light of these data, Ire1 $\alpha$ loss-mediated increase in polysome level may represent stalled ribosomes, accumulated in the polysome fraction. 

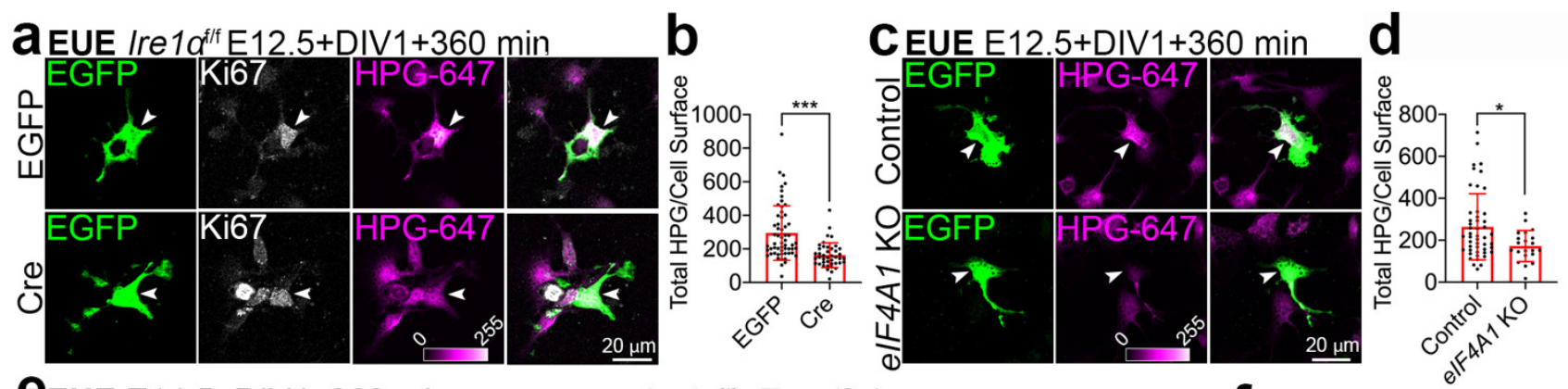

405

$\mathbf{e}_{\text {EUE E14.5+DIV4+360 min }}$

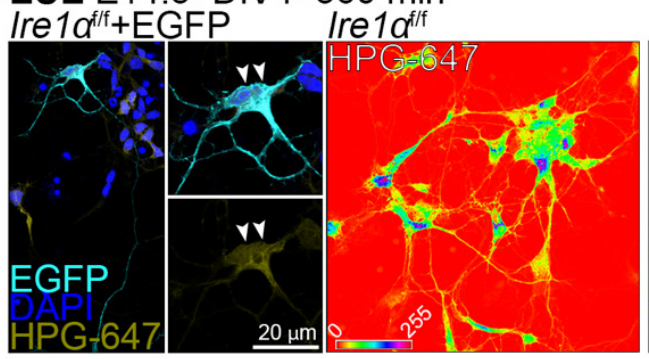

Ire $1 \alpha^{\text {flf; }}$ Emx $1^{\text {Crel+ }}$

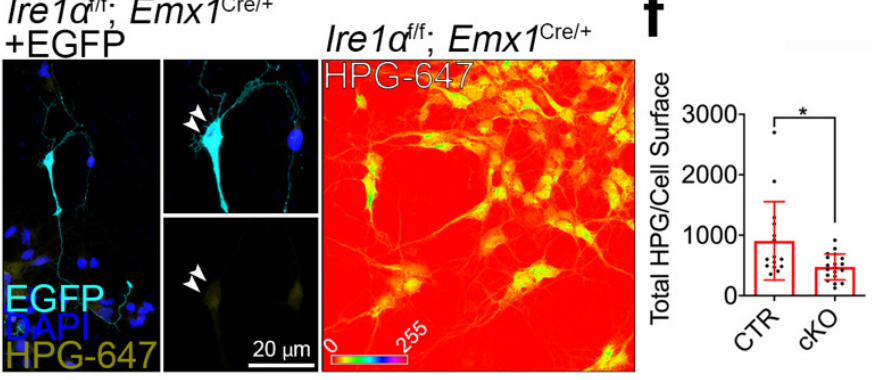

Fig. 4. Loss of Ire1a results in lower translation rates.

(a), (c), (e) Images of representative primary cortical neurons prepared from Ire $1 \alpha^{\text {f/f }}$ (a), wild type (c) or control and Ire1 $\alpha$ cKO (e) embryos after EUE at E12.5 (a and c) or E14.5 (e) with indicated plasmids. Neurons were fed L-homopropargylglycine (HPG) for 360 min prior to fixation at DIV1 or

410 DIV4. HPG was detected with Sulfo-Cyanine5 azide. (e) Right panels: images of HPG incorporation in control and cKO primary DIV4 neurons prepared from E14.5 cortex. White arrowheads point to Ki67-positive progenitors (a and c) or to somata of neurons derived from E14.5 progenitors (e). (b), (d), (f) Quantification of HPG incorporation. Bar graph represent data points for every analyzed cell and averages \pm S.D. Statistics for (b), (d), and (f), D'Agostino-Pearson normality test and Mann415 Whitney test; $0.01<{ }^{*} p<0.05$; ${ }^{* *} p<0.001$. 


\section{Deeper layer cortical neurons exhibit higher translation rates than upper layer neurons}

Given lower translation rates in Ire1a cKO neurons (Fig. 4), and our findings of Ire1amediated specification of neuronal subtype (Fig. 1), we next asked whether differences in

420 translation rates are an intrinsic feature of neurons of different cortical layers.

In order to test this, on the same day we nucleofected two populations of e12 and e14 primary cortical cells with dsRed and EGFP-expressing plasmids, respectively, mixed the now differentially labeled cells, and cultured together to tightly control for the microenvironment (to separate these sets of experiments from ones performed on Ire1 $\alpha$, we

425 labeled the embryonic stages with a lowercase "e"). We then fixed the cells at DIV1 and DIV5, following an HPG pulse of varying duration (Fig. 5). First, we validated what cell types are present at these two culture stages using immunostaining against proliferative markers Ki67 and Pax6, and postmitotic identity markers (Fig. 5a and 5b). Our nucleofection and culture system enriches for deeper layer marker-expressing neurons derived from e12 cortex, for

430 Satb2 in neurons prepared from e14 cortex, and generally for postmitotic neurons (Fig. 5b), with only a minor fraction of cycling cells or radial progenitors still present at DIV1 (Fig. 5c). We pulsed HPG in the methionine-free cell culture medium for 60, 120 and 240 minutes at DIV1 (for immature neurons) and at DIV5 (Fig. 4d). At DIV1, following HPG application, we quantified significantly higher HPG incorporation level in e12- (dsRed+) versus e14- (EGFP+)

435 derived neurons (Fig. 5e and 5f). We also detected higher HPG incorporation rates at DIV5, with a milder difference between the two cell populations. Secondly, we observed, that in both e12- and e14-derived neuronal cultures, HPG incorporation rates were higher at DIV1 when compared to DIV5 (Fig. 5e and 5g). The addition of cycloheximide (CHX), a translation inhibitor (Schneider-Poetsch et al., 2010), attenuates HPG incorporation (Fig. 5h), reinforcing

440 the translation-specific nature of our findings. Altogether, these data indicate that deep layerfated neurons have inherently higher translation rates compared to upper layer neurons. 


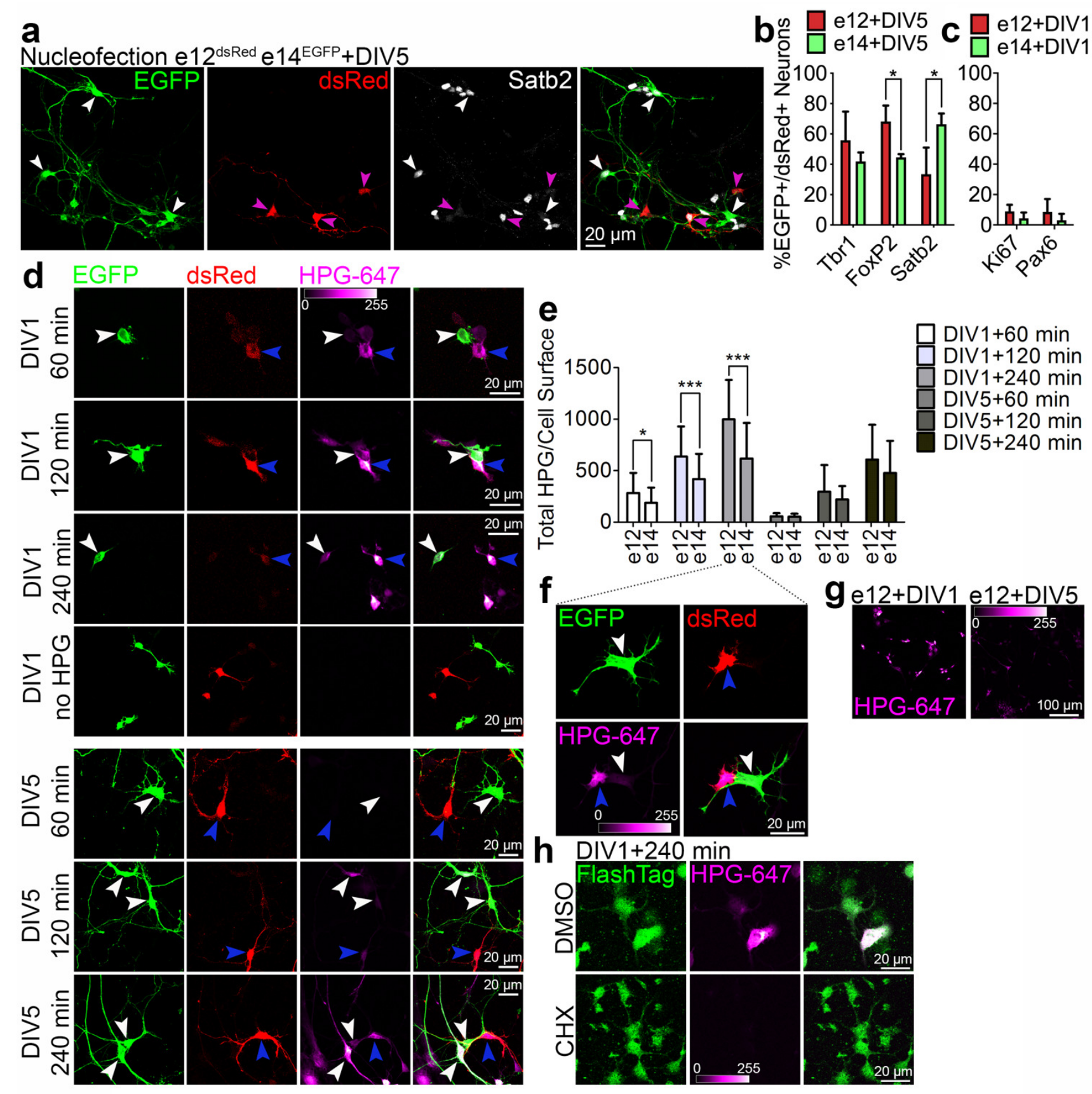

Fig. 5. Increased translation rate is a hallmark of early born, deeper layer postmitotic neurons 445 in the developing cortex. (a) Images of primary DIV5 neurons immunolabeled for EGFP, dsRed, and Satb2. Cortical cells from e12 embryo were nucleofected to express dsRed (purple arrowheads), and cortical cells from e14 embryo to express EGFP (white arrowheads). Both cell populations were mixed together and plated on a single glass coverslip. (b, c) Quantification of the cell identity in DIV5 neurons (b) and DIV1 primary cells (c) derived from e12 or e14 cortex. (d) Images of primary cortical 450 cells immunolabeled for EGFP (white arrowheads) and dsRed (blue arrowheads), pulsed with HPG for indicated amount of time before fixation at DIV1 and DIV5. HPG was detected with far red fluorophore-coupled azide. (e) Incorporation of HPG was quantified as intensity of fluorescence signal normalized to the cell surface in primary neurons. (f) DIV1 primary cortical cells from (a), fed with HPG for 240 min. Note significantly higher HPG uptake in e12 cortex-derived immature neurons. (g) HPG

455 labeling in primary neurons at DIV1 and DIV5 prepared from e12 cortex. Note significantly higher HPG uptake in primary cultures at DIV1. (h) DIV1 primary cortical cells from e14 cortex, fed with HPG 
bioRxiv preprint doi: https://doi.org/10.1101/2021.06.23.449563; this version posted June 23, 2021. The copyright holder for this preprint (which was not certified by peer review) is the author/funder. All rights reserved. No reuse allowed without permission.

for $240 \mathrm{~min}$ in presence of DMSO or $10 \mu \mathrm{g} / \mathrm{mL}$ cycloheximide (CHX). Note strongly reduced HPG labeling in cells cultured with $\mathrm{CHX}$. FlashTag was added to the medium to label all cells. Bar graphs indicate mean \pm S.D. Statistics for (b) and (c), D'Agostino-Pearson normality test and Mann-Whitney 460 or unpaired t-test; (e), two-way ANOVA with Bonferroni post-tests. ${ }^{* *} p<0.001 ; 0.01<{ }^{*} p<0.05$. 
The transition from multipotent neuronal progenitors to progenitors of upper layer neurons is associated with a pronounced upregulation of translation

Next, we studied the global translation rates in cycling progenitors of deeper and upper layer

465 neurons and in their directly derived progeny. To address this, we used EUE, which unlike the nucleofection (Fig. 5) allows for labeling cycling progenitors. We transfected ventricular e12 progenitors with dsRed- and e14 progenitors with EGFP-encoding vectors, then cultured the cells until DIV1 and DIV5, with HPG-feeding prior to fixation. We then quantified HPG incorporation in Ki67-labeled DIV1 progenitors and in DIV5 postmitotic neurons (Fig. 6a) and

470 found that e14 upper layer progenitors display profoundly higher translation rates as compared to e12 multipotent progenitors. Additionally, consistently with results shown in Fig. 5, e12 progenitor-derived postmitotic neurons display higher HPG incorporation rates as compared to e14 progenitor-derived cells. Translation rates undergo a significant upregulation in e14 cortical progenitors that dramatically decreases during postmitotic

475 differentiation of an upper layer neuron (Fig. 6b). The majority of e12 progenitor-derived postmitotic cells co-express both Satb2 and CTIP2 in culture, and e14 progenitor-derived ones express Satb2, but not CTIP2 (Fig. 6c and 6d).

Taken together, the translation rate is a dynamic feature of cortical progenitors and their derived progeny, and likely represents cell- and stage-specific requirements of different 480 protein sets during development. 


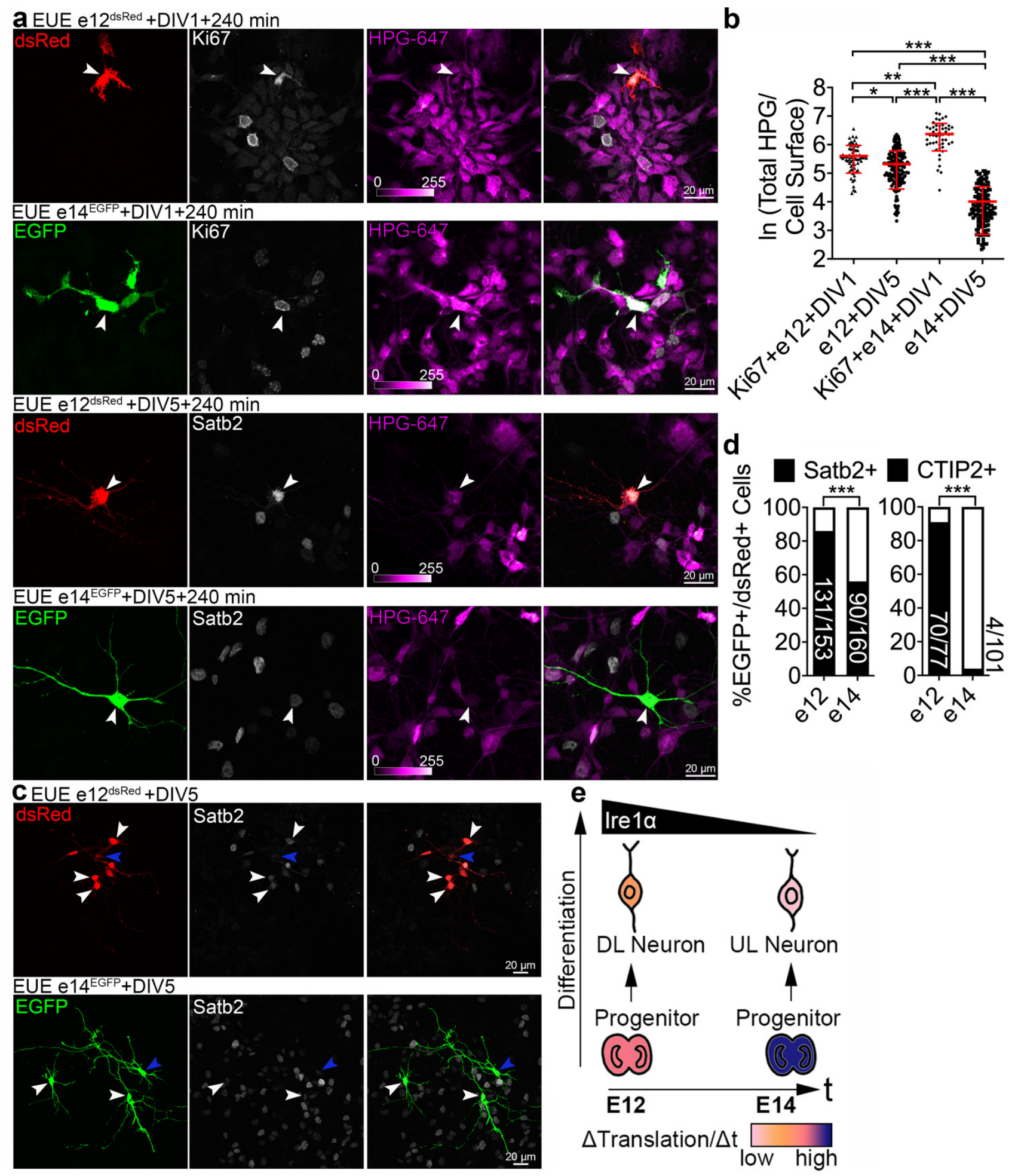

Fig. 6. Progenitors of upper layers display dramatically higher translation rates than progenitors of deeper layers.

485 (a) Images of primary DIV1 and DIV5 cortical cells immunolabeled for EGFP, dsRed, Ki67, and Satb2. To target Ki67-positive neuronal progenitors and their derived progeny, cortices of e12 embryos were ex utero electroporated (EUE) to express dsRed, and cortices of e14 embryos to express EGFP. Cells were triturated, mixed, plated together on a glass coverslip and pulsed with HPG for 240 min prior to fixation at DIV1 and DIV5. White arrowheads indicate representative cells. (b) Incorporation of HPG 490 was quantified as intensity of fluorescence signal normalized to the cell surface area (natural logarithm scale). (c) e12 and e14-derived cells were fixed at DIV5 and immunolabeled for Satb2. White 
arrowheads point to neurons expressing high levels of Satb2, blue ones indicate example neurons with lower Satb2 expression. (d) Quantification of the cell identity markers in DIV5 neurons. (e) Schematic summarizing the translation rates $(\Delta$ Translation/ $\Delta t)$ in different types of cortical cells for 495 e12- and e14-derived lineage. The triangles indicate the dynamics of developmental enrichment for Ire1 $\alpha$; deeper layers (DL) and upper layers (UL). Graph on (b) indicates mean \pm S.D. Statistics for (b), D'Agostino-Pearson normality test and Kruskal-Wallis test with Dunn's multiple comparisons test; (d), Fisher's test. ${ }^{* * *} p<0.001 ; 0.001<{ }^{* *} p<0.01 ; 0.01<{ }^{*} p<0.05$. 


\section{Transient attenuation of translation results in loss of axon and upper layer type identity}

We then asked if pharmacologically attenuating translation early in development alters the specification of upper layer type neurons. Given a significant difference in translation rates at DIV1 between e12- and e14-derived neuronal cultures, we nucleofected the primary cells to express dsRed or EGFP as described above and exposed them to DMSO or cycloheximide

$505(\mathrm{CHX})$, an inhibitor of protein synthesis, immediately after plating transiently for 24 hours. Following this, we changed the cell culture medium to one lacking $\mathrm{CHX}$ and maintained the primary neuronal cultures until DIV5 (Fig. 7a). Remarkably, transient inhibition of translation affected the appearance of Satb2 expression in cells derived from both stages but had no effect on the expression of CTIP2 (Fig. 7b).

$510 \quad$ Additionally, neurons transiently exposed to $\mathrm{CHX}$ failed to project a long neurite at DIV5 (Fig. S5a). While control EGFP-expressing upper layer neurons displayed an asymmetric morphology with a longest neurite enriched for Tau-1 (Fig. S5a), most neurons transiently exposed to $\mathrm{CHX}$ failed to break their symmetry and expressed Tau- 1 in the perinuclear regions of soma, resembling the scenario upon Ire1a (Fig. S3a, bottom panels).

515 We quantified a CHX-induced dose-dependent loss of axons (Fig. S5b) and overall loss of neurite branching (Fig. S5c and S5d).

Altogether, transiently inhibiting protein synthesis at early developmental stages leads to irrevocable loss of Satb2 identity and loss of axons, indicative of critical translational window for both processes and reminiscent of Ire1 $\alpha \mathrm{KO}$. 


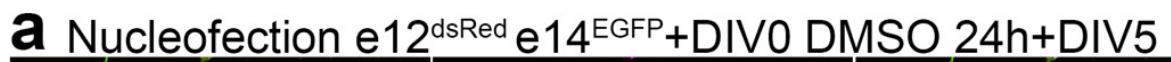

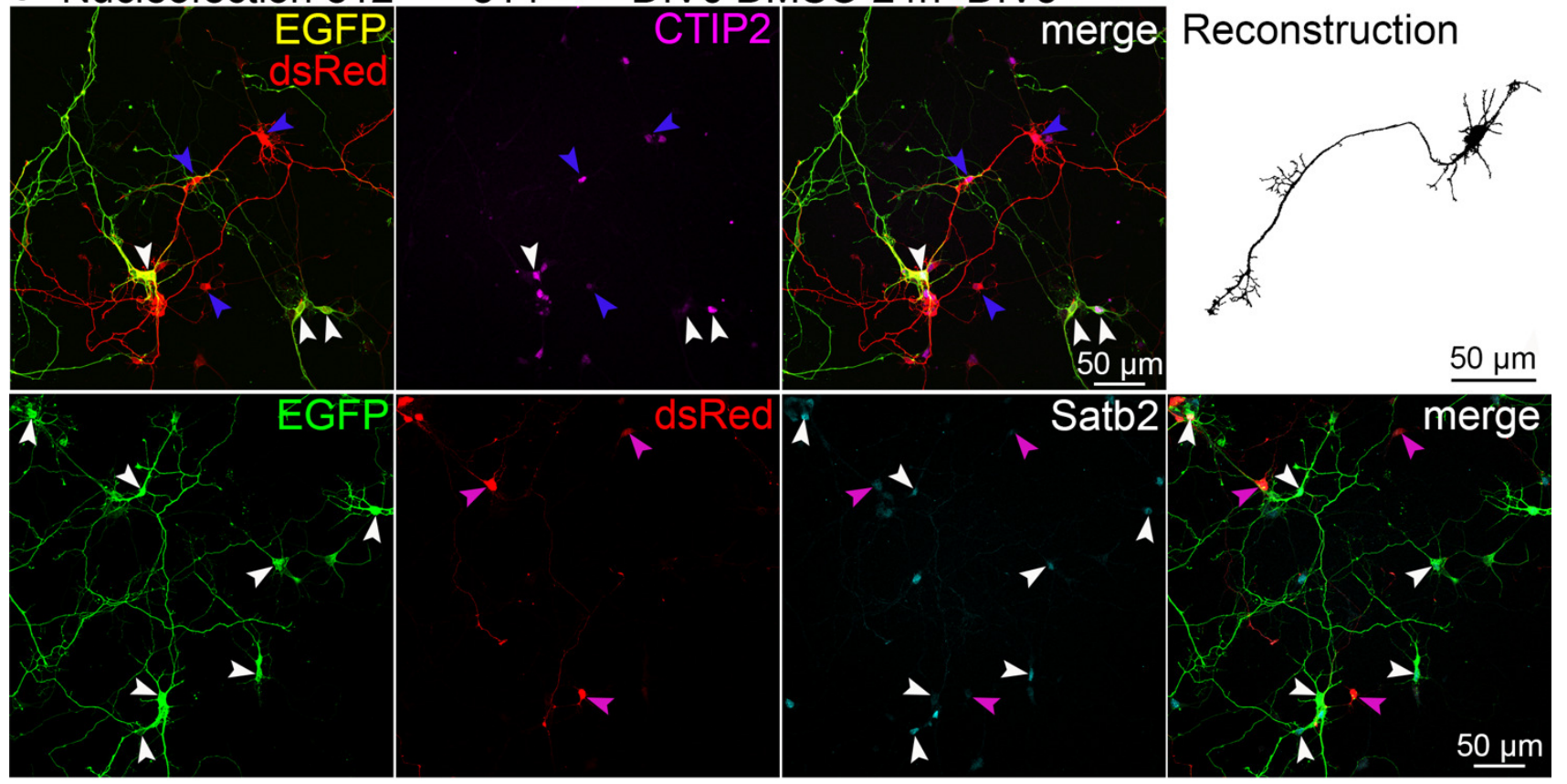

Nucleofection e12 ${ }^{\text {dsRed }}$ e14 ${ }^{\text {EGFP }}+$ DIVO CHX $10 \mu \mathrm{g} / \mathrm{mL} 24 \mathrm{~h}+\mathrm{DIV} 5$
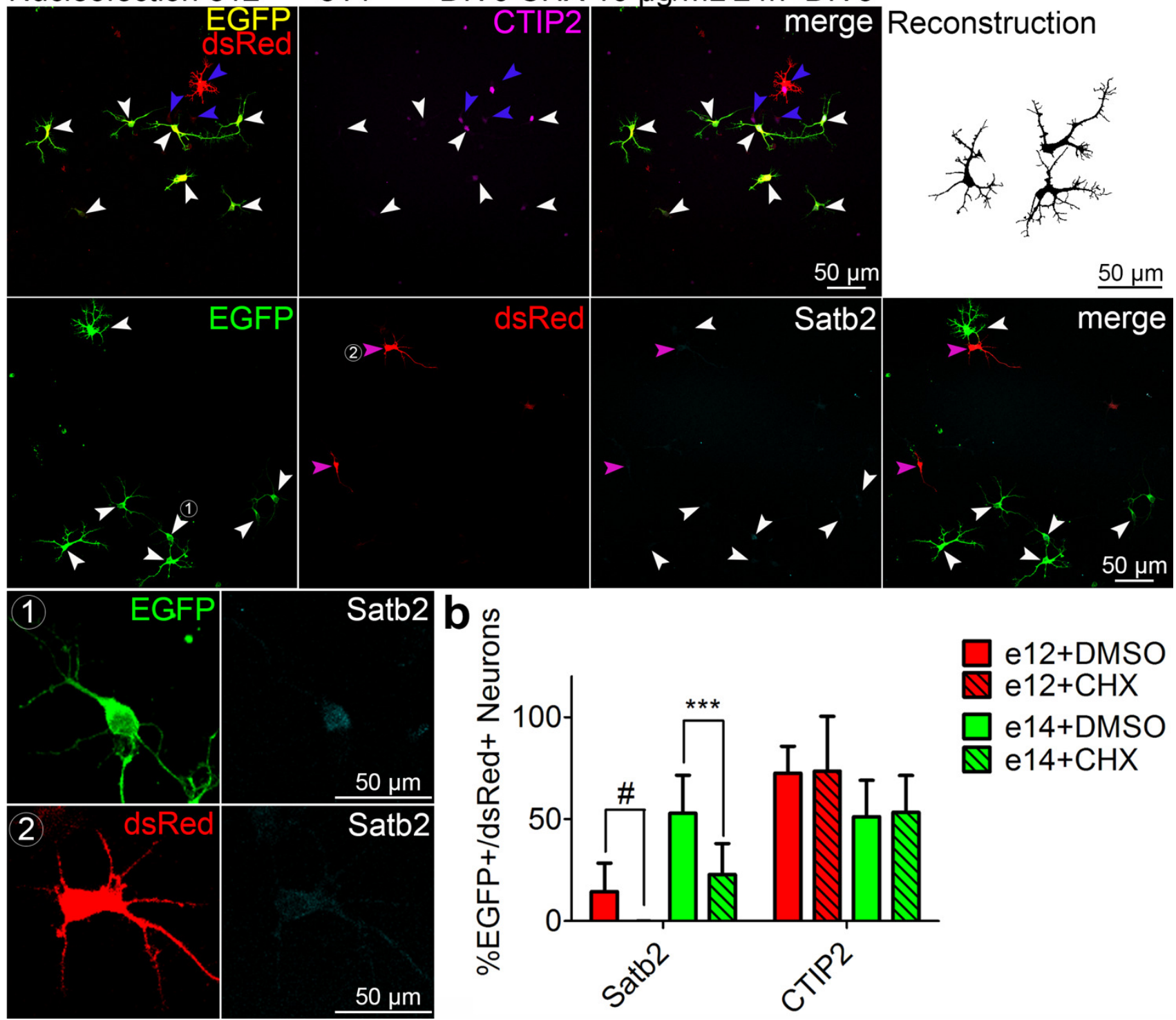
Fig. 7. Upper layer identity requires a critical window of protein translation in precursor cells. (a) Images of immunolabeled primary cells from e12 embryo nucleofected to express dsRed, and from e14 embryo to express EGFP, mixed and plated on a single glass coverslip. Two hours post525 plating, cells were treated with vehicle (DMSO) or cycloheximide ( $\mathrm{CHX}$ ) for 20 hours, followed by medium change, and fixed at DIV5. Upper panels show staining using rat anti-CTIP2, goat anti-EGFP, and rabbit anti-RFP, the latter one recognizing both EGFP and dsRed. For this reason, e12 cells in this case were recognized as solely expressing dsRed (blue arrowheads), but the e14 ones, both EGFP and dsRed (white arrowheads). Lower panels show anti-Satb2, anti-EGFP and anti-dsRed 530 immunostaining with no cross-reacting antibodies; in this case, e12-derived cells express dsRed (blue arrowheads) and e14 ones EGFP (white arrowheads), as expected. Representative neuronal morphology is demonstrated as a semi-automated, EGFP- or dsRed-based reconstruction. (1-2) Example cycloheximide-treated e14 (1) or e12 (2) cortex-derived cells immunolabeled with an antibody anti-Satb2. (b) Quantification of the cell identity markers in DIV5 neurons derived from e12 535 or e14 cortex. Bar graphs indicate mean \pm S.D. For statistics, two-way ANOVA with Bonferroni's multiple comparisons test. \# indicates a comparison between fractions of Satb2-positive e12 DMSOand e12 CHX-treated group, the latter represented by virtually no positive cell. ${ }^{* *} p<0.001$. 


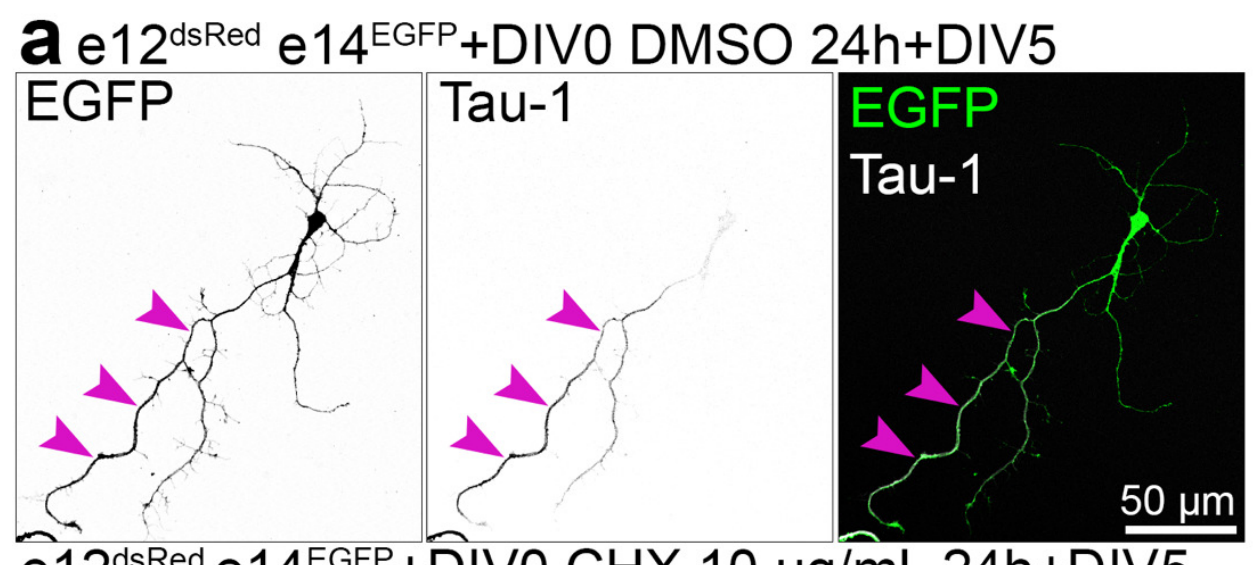

$\widehat{\mathrm{e}} 12^{\text {dsRed }}$ e14 ${ }^{\mathrm{EGFP}}+\widehat{\mathrm{DIV}} \mathrm{CHX} 10 \mu \mathrm{g} / \mathrm{mL}$ 24h+DIV5
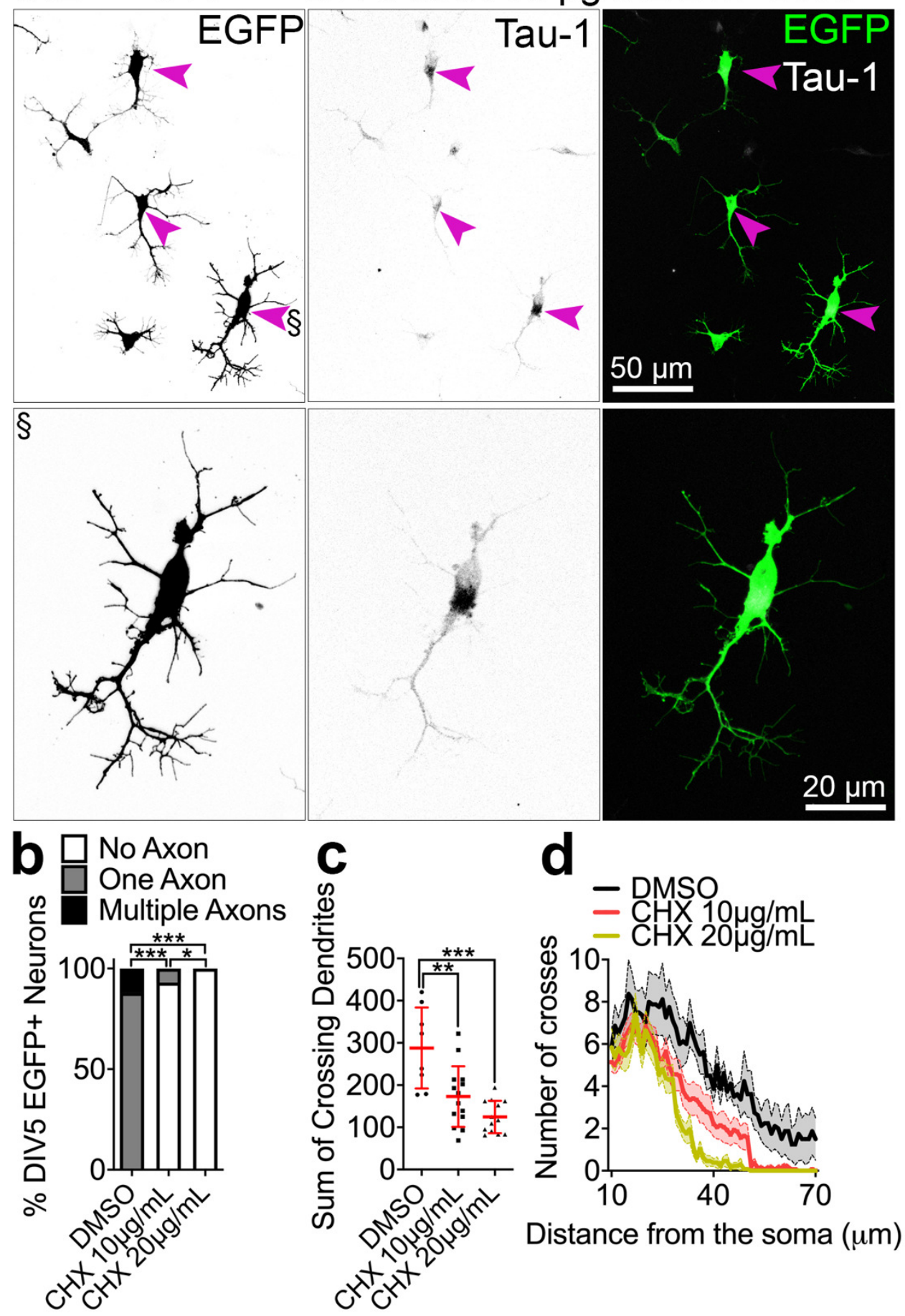

Distance from the soma $(\mu \mathrm{m})$ $c^{x+c^{2}}$ 
540 Fig. S5. Related to Fig. 7. Axon formation and dendritic growth requires a critical window of translation in precursor cells.

(a) Images of immunolabeled primary cells prepared as described in the legend of Fig. 7a. Cells were fixed at DIV5 and immunolabeled for EGFP, dsRed and Tau-1. Purple arrowheads indicate enrichments of Tau-1. The neuron marked with a paragraph symbol is magnified on the bottom panel.

545 (b) Quantification of neuronal polarity. Axon was defined as the neurite with prominent Tau-1 enrichment [compare with top panel in (a)]. (c-d) Quantification of dendritic complexity in DMSO-and CHX-treated DIV5 neurons. Red line and error bars on (c) indicate mean \pm S.D. Results on (d) are represented as averages \pm S.E.M. For statistical analyses, (b), Chi-square test; (c), D’Agostino and Pearson normality test and one-way ANOVA with Bonferroni post-hoc test. ${ }^{* * *} p<0.001 ; 0.001<{ }^{* *}$ $550 \mathrm{p}<0.01 ; 0.01<{ }^{*} p<0.05$. 
aFig. 1e. IUE E12.5 - E16.5 Single Plane

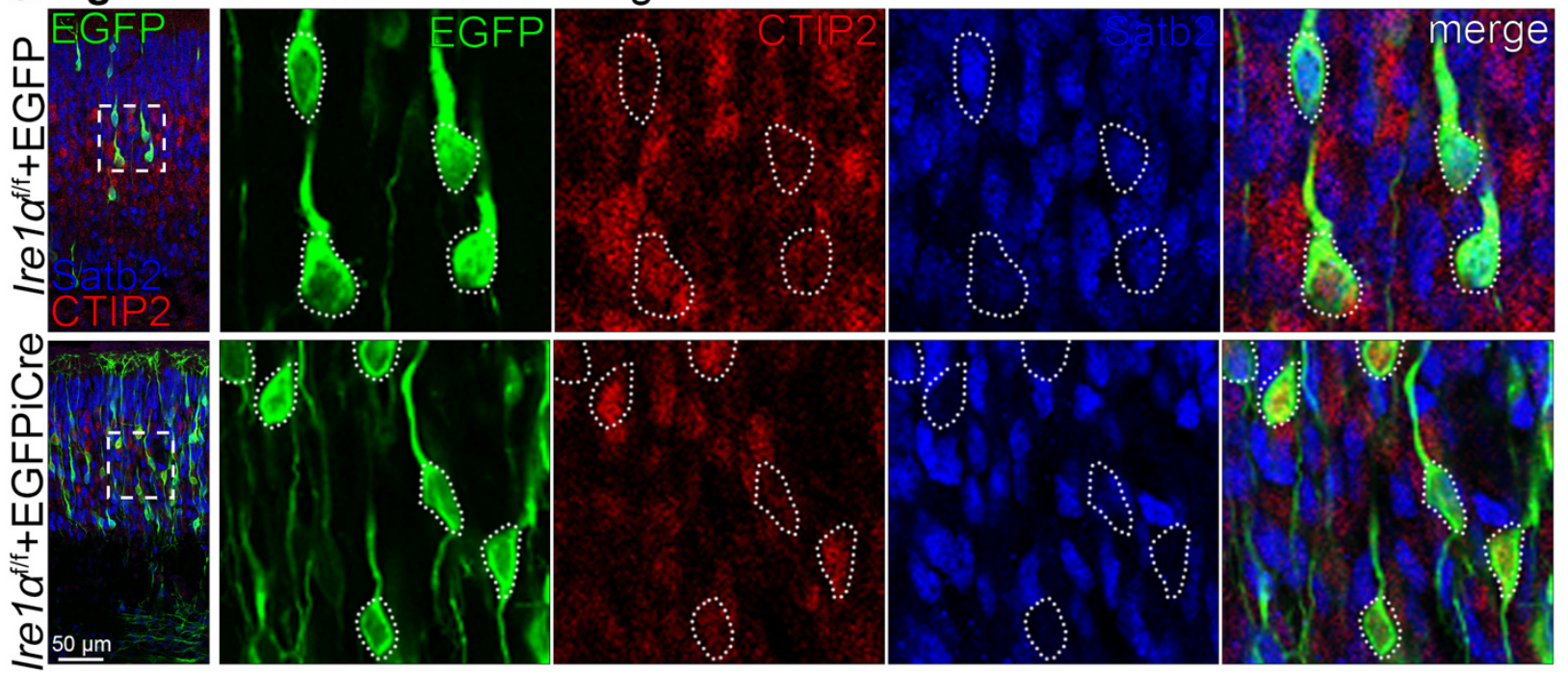

bFig. 1h. IUE E12.5 - E18.5 Max Intensity Projection

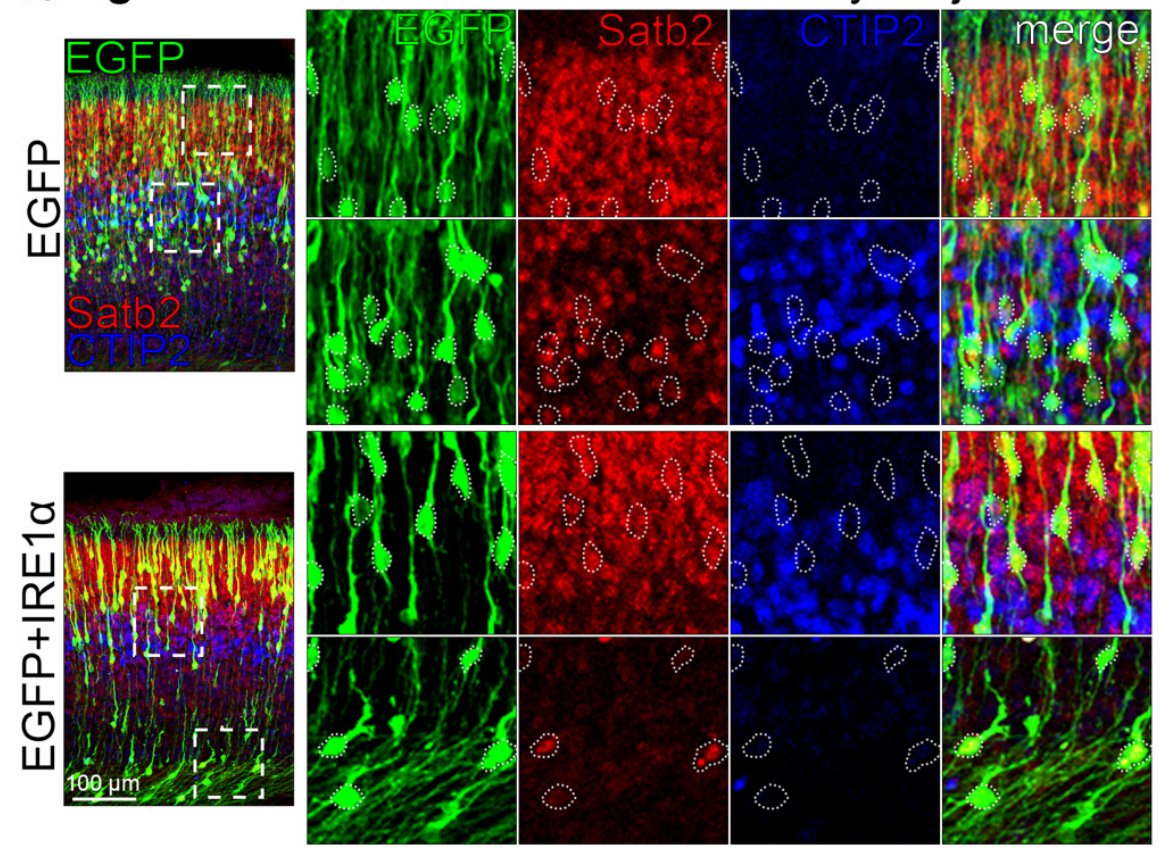

C Fig. 3g. IUE E12.5 - E18.5 Single Optical Plane

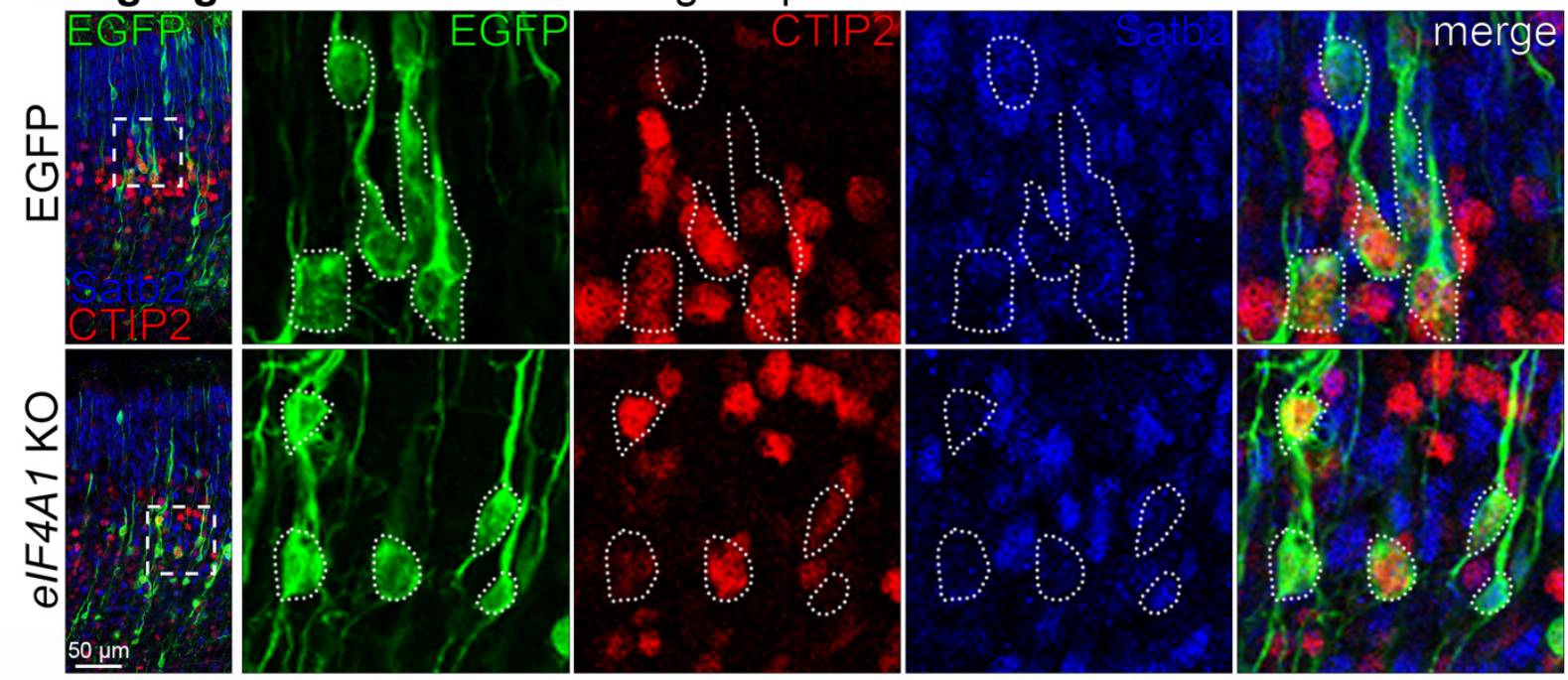


555 Fig. S6. Related to Fig. 1, Fig. 3. Zoom-ins for the neuronal identity phenotypes.

(a-c) Representative images of immunostaining against EGFP, Satb2, and CTIP2 in coronal cortical sections after IUE at E12.5 for indicated conditions. Each panel represents a sample image pair used for identity analysis in experiment indicated on top. The squares depict the area of the zoomin, outlined are somata of electroporated neurons. Neuronal identity was quantified in the proportion

560 of all electroporated neurons in a given coronal section, here shown are snippets for a visualization of immunostaining. 


\section{DISCUSSION}

Development of the cortex comprises an orchestrated series of sequential, tightly controlled gene expression events with many layers of regulation. Our data implicate the regulation of protein synthesis rate by Ire1a in the specification of upper layer neurons and their polarization. Through identifying a developmental role for Ire1 $\alpha$ beyond its canonical role in

570 translation stress pathways, we make the surprising finding that different protein synthesis rates are intrinsic features of distinct progenitor and differentiated neuron lineages.

The demand to swiftly and efficiently synthesize and remodel the proteome during cortical development places homeostatic pathways on the brink of cellular stress signaling. We speculate that such demands in normal development are met by the same molecular

575 players as stress pathways, however, likely leading to unique downstream pathways active in development, such as a Satb2 program. During cortical development, Ire1a supervises the cellular translation flux (Fig. 4) by driving the expression of translation regulators and RNA binding proteins, essential for the high protein synthesis rate in upper layer progenitors, enabling translation of Satb2 in their postmitotic progeny (Fig. 3) and proteins crucial for

580 specification of an axon. By labeling the amount of HPG incorporated into the newly synthesized proteins in a given time period, we quantify the total translation flux in a cell. HPG incorporation reflects several translation-regulating processes, e.g. the translation efficiency, polypeptide chain elongation, codon dwell times, overall representing the rate of protein synthesis (Fig. 4).

585 Seemingly, on top of elegant transcriptional regulation (Nowakowski et al., 2017; Telley et al., 2019), generation of upper layer neurons relies on specific pathways for translational control. One possible scenario involves specific features of 5'UTR regions in genes critical for upper layers (Hinnebusch et al., 2016) and activity of translation initiation complexes, comprising elF4A, which unwind cap-proximal regions of mRNA prior to its

590 ribosomal loading (Andersen, 2006). In line with this are our observations on decreased translation rates upon eIF4A1 KO (Fig. 4c and 4d) associated with defects in upper layer neurogenesis (Fig. $3 g$ and $3 \mathrm{~h}$ ).

The Ire1 $\alpha$ cKO partially mimics the Satb2 KO (Britanova et al., 2008), with the loss of upper layer type neurons at the expense of CTIP2-expressing deeper layer cells (Fig. 1 and

595 S2). Strikingly, in the Ire1 $\alpha$ cKO cortex CTIP2-expressing neurons are specified during late neurogenesis (Fig. S2e-S2h). Wild type neuronal progenitors at e12.5 generate CTIP2 and Satb2 positive cells, and as development progresses, late progenitors give rise to Satb2expressing progeny (Fig. S2 and 5-6). Such progressive restriction of neuronal progenitor potency is abolished upon Ire1 $\alpha$ loss-of-function. In contrast, high activity of IRE1 $\alpha$ correlates

600 with lower expression of stemness markers, like SOX2, POU3F2 or OLIG2 (Doultsinos et al., 2019).

It is worth emphasizing the results in Fig. 5 and 6 represent different ways of lineage labeling (bulk nucleofection versus clonal progenitor tree), which accounts for different fate signatures in the postmitotic progeny. Our approach to mix labeled cells derived from

605 embryonic cortices of different developmental stages allows for exposure to identical culturing conditions. An interesting future direction is to examine how cellular and environmental composition affects the identity of progenitors, neurons, and associated translation rates. 
Global attenuation of translation during the UPR is linked to PERK-mediated phosphorylation of elF2 $\alpha$ at Ser51 (Krishnamoorthy et al., 2001). The stress response alters 610 the cellular translation machinery and its affinity towards open reading frames of specific type, position, and secondary structures (Starck et al., 2016; Vattem and Wek, 2004). We report that loss of Ire $1 \alpha$ results in lower rates of protein synthesis in neuronal progenitors and neurons, independently of elF2 $\alpha$, or its canonical downstream splicing client Xbp1 (Fig. S4a and S4b). Ire1 $\alpha$ regulates the amount of translating polysomes, with higher levels in the cKO.

615 In the context of lower translation flux, this likely represents ribosome stalling and/or slower elongation, reminiscent of observations in Huntingtin mutants (Eshraghi et al., 2021). As Ire1a binds $80 \mathrm{~S}$ ribosomes directly with high affinity (Acosta-Alvear et al., 2018), and is in the vicinity of the ribosomes embedded in the ER by the translocon, it is well positioned to impact protein synthesis directly during cortex development.

$620 \quad$ Protein synthesis inhibition with $\mathrm{CHX}$ resembles the Ire1 $\alpha$ cKO and eIF4A1 KO (albeit engendering expected stronger phenotypes, given the global inhibition of translation by the compound), with defects in Satb2 expression and axonal targeting of Tau-1, leading to the formation of symmetrical, non-polarized neurons and ablation of Satb2 (Fig. 7 and S6). Both the loss of Ire1 $\alpha$ and $\mathrm{CHX}$ spare the expression and subcellular localization of deep layer

625 neurons-associated CTIP2 and dendrite-enriched MAP2, indicative of the specific requirement for Ire1 $\alpha$ and high translation rates during upper layer neurogenesis and polarization. In line with this, we find that late neuronal progenitors display a drastic increase in translation rates.

Apart from being a site of protein synthesis, the ER has been also reported as an

630 organelle crucial for neuronal polarization (Farías et al., 2019). Upon Ire1a KO, we report a mislocalization of the ER to the polarizing neurite (Fig. $2 m$ and $2 n$ ). Defects in axon specification, and thus axonal targeting of Tau-1, AnkG and $\mathrm{Na}_{\mathrm{v}}$ (Fig. S3) might indicate ER dysfunction upon Ire1a loss, implicating its role in ER integrity and neuronal polarization (Carter et al., 2021). Loss of polarization might also partially explain the filamin A-dependent

635 Ire1 $\alpha$ loss-of-function phenotype in laminar positioning of cortical neurons (Urra et al., 2018). Emergence of either multiple axons or more commonly no axons in Ire $1 \alpha \mathrm{KO}$ neurons (Fig. 2) implies a disruption of distinct axon polarity-regulating processes in this mutant, likely involving cellular sorting of axonal proteins and ER-dependent microtubule stability, overall leading to disturbed transmission of action potentials. Cortical neuronal migration and

640 neuronal polarization involves Ire1 $\alpha$-driven control of multiple downstream processes, filamin A anchoring on one hand, eEF-2- and elF4A1-regulated translation on the other hand.

Taken together, our study reinforces the powerful impact of post-transcriptional mechanisms in cortex development. Translational regulation of gene expression during neuronal specification is layered on top of transcriptional mechanisms, many of which have

645 been highlighted in excellent recent work (Nowakowski et al., 2017; Telley et al., 2019). Modulation of translation rates may be a particular requirement for upper layer specification and morphology, evolving as a mechanism to specify upper layers in the late stages of development while restricting the potential of neural progenitors to this lineage. We speculate that a swift upregulation of translation flux in late progenitors might be necessary to efficiently 650 synthesize determinants of upper layer neurons. In the light of elF4A1 requirement for Satb2 
identity, it is possible that 5'UTR-embedded structural features are present in mRNAs critical for differentiation of upper layer neurons. Given developmental downregulation of Ire1 $\alpha$, it is plausible that other translational regulators alongside Ire1 $\alpha$ drive the increased translation efficiency in upper layer progenitors. Thus, translational control by in part by Ire1 $\alpha$ is deployed

655 in normal development to promote upper layer neurogenesis, generate their axons, and wire the mammalian cortex.

\section{SOURCE DATA}

Sequencing data are available on NCBI under GSE172489. 660

\section{ACKNOWLEDGEMENTS}

This work was supported by the German Research Foundation (TA 303/15-1) and the Russian Scientific Foundation (21-65-00017). We thank Mengfei Gao, Helge Ewers, the organizers of CAJAL Advanced Imaging Methods Course for Cellular Neuroscience and

665 Boehringer Ingelheim Fonds for the introduction to expansion microscopy. We thank Paraskevi Bessa-Newman for her useful tips during the initial phase of the project and Hiroshi Kawabe for the pRai plasmid.

\section{AUTHOR CONTRIBUTIONS}

670 M.C.A. carried out and conceptualized the study, with significant contribution from E.B. V.T. designed the inhibitor screening, initiated, and supervised the project. A. G. N. performed the experiments with RNAseq and the bioinformatics. M. L. K. purified the ribosomes ex-vivo and analyzed their profiles. T. S. performed the molecular cloning. R. D. significantly contributed to histological and cell culture experiments. M. B. recorded from autaptic cultures and

675 analyzed the results. P. T. shared his expertise and protocols for the FUNCAT and click chemistry. D. K. helped with the initial screening and share the molecule library. O. J. performed proteomic analyses. T. I. shared the $I r e 1 a^{f / f}$ mouse line. M. R., C. M. T. S., and C. R. supervised works in their labs.

\section{CONFLICT OF INTEREST}

The authors declare no competing interests.

\section{STAR METHODS}

\section{CONTACT FOR REAGENTS AND RESOURCE SHARING}

685 Further information and requests for resources and reagents should be directed to Victor Tarabykin (victor.tarabykin@charite.de). Detailed information on the materials used in this study can be found in Key Resource Table.

\section{EXPERIMENTAL MATERIAL AND SUBJECT DETAILS}

690 Animals

Mouse (Mus musculus) lines described in this study have been maintained in the animal facilities of the the Charité University Hospital and Lobachevsky State University. Wild type mice were of NMRI strain. In Ire $1 \alpha^{\mathrm{f} / \mathrm{f}}$ mouse line (Iwawaki et al., 2009), exons 20-21 of Ire1a were engineered to be flanked by loxP sites. To inactivate Ire1 $\alpha$ in the developing cortex, we 
695 crossed $I r e 1 \alpha^{f / f}$ mice with the $E m x 1^{C r e /+}$ line, in which Cre recombinase is expressed from the Emx1 gene allele (Gorski et al., 2002). As described in our previous work, for the breeding we exclusively used Ire $1 \alpha^{\mathrm{flf}}$ males and Ire $1 \alpha^{\mathrm{f} / \mathrm{f}} ; \mathrm{Em} \times 1^{\mathrm{Cre} /+}$ females to circumvent leaky expression of Cre (Luo et al., 2020). Animals homozygous for the loxP alleles in both lines were viable, fertile, and born at the expected Mendelian ratio, and exhibited no overt

700 phenotypic changes in the cage environment. For experiments with tdTomato reporter (Fig. 1a-1b), Satb2 ${ }^{\mathrm{Cre} /+}$ (Britanova et al., 2006) males were mated to NMRI wild type females. The date of vaginal plug was counted as E0.5. All mice were sacrificed by administering a lethal dose of pentobarbital.

All experiments were performed in compliance with the guidelines for the welfare of

705 experimental animals approved by the State Office for Health and Social Affairs, Council in Berlin, Landesamt für Gesundheit und Soziales (LaGeSo), permissions G0079/11, G206/16, and G54/19, and by the Ethical Committee of the Lobachevsky State University of Nizhny Novgorod.

\section{Sex and age/developmental stage of animals for in vivo experiments}

Littermates of both sexes were randomly assigned to experimental groups during experimental procedures or collection of embryonic tissue. Developmental stages or stages at experimental interventions are listed on the figures or in the figure legends.

\section{Murine primary neurons}

Embryonic brains were dissected on ice-cold Hank's Balanced Salt Solution (HBSS) under stereomicroscope to collect cortices in $5 \mathrm{~mL}$ of ice-cold $0.25 \%$ trypsin (Gibco) solution. After incubation for $20 \mathrm{~min}$ at $37^{\circ} \mathrm{C}$ with occasional swirling, digestion was terminated by addition of $1 \mathrm{~mL}$ of fetal bovine serum (FBS, Gibco) and $1 \mathrm{U}$ of DNasel (Roche) was added to the tube

720 for $1 \mathrm{~min}$. Next, cortices were carefully washed twice with $5 \mathrm{~mL}$ of pre-warmed $\left(37^{\circ} \mathrm{C}\right)$ Complete Neurobasal medium. Further, cortices were carefully triturated in $1 \mathrm{~mL}$ Complete Neurobasal medium 10 times using P200 pipette tip. The debris were then let sink for 1 minute and $150 \mu \mathrm{L}$ the supernatant transferred to $900 \mu \mathrm{L}$ of fresh Complete Neurobasal at $37^{\circ} \mathrm{C}$. After second round of trituration, cells were counted with Naubauer counting chamber

725 and neurons were seeded in 24 well-plate formats at 120000 cells (EUE) or 240000 cells (after nucleofection) per coverslip in $1 \mathrm{~mL}$ of Complete Neurobasal medium. The day of neuronal prep was counted as day in vitro 0 (DIV0). Neurons were cultivated at $37^{\circ} \mathrm{C}$ in the presence of $5 \%$ carbon dioxide in HERA-cell240 (Heraeus) incubator. For the experiments with DMSO and $\mathrm{CHX}$, neuronal cultures were treated with the substance two hours post-

730 plating. Given substance was thoroughly mixed with fresh medium and placed on attached cells.

Autaptic hippocampal cultures were prepared from P0 Ire $1 \alpha^{f / f}$ mice as described (Giusti et al., 2014).

735 Complete Neurobasal: 500 mL Neurobasal (Gibco, Life Technologies), 10 mL B-27 (Gibco, Life Technologies), $5 \mathrm{~mL}$ GlutaMAX (Gibco, Life Technologies), $5 \mathrm{~mL}$ penicillin/streptomycin (Life Technologies, Gibco), $1 \mathrm{~mL}$ Primocin (Invitrogen). 


\section{METHODS}

\section{Lentivirus production and infection of autaptic hippocampal cells}

Production of lentiviral particles and infection of autaptic cultures was performed by the Charité Viral Core Facility according to standard published protocols.

\section{Electrophysiological Recordings}

745 Voltage and current clamp experiments were performed as described before (Giusti et al., 2014).

\section{Expression vectors}

For our screening, we used beta-actin driven expression constructs pCAG-EGFP and pCAG-

750 flox-stop-flox-tdTomato, which are described in our previous work in detail (Ambrozkiewicz et al., 2017)

\section{Cloning strategies for constructs generated in this study}

Cloning of all expression vectors in this study was performed using the NEBuilder system

755 according to manufacturer's protocol (New England BioLabs). DNA fragments were amplified using GXL Prime Star DNA polymerase (Takara) using cDNA libraries as templates. Destination vectors were linearized with EcoRI-HF (New England BioLabs).

\section{pRai-HA-IRE1 $\alpha$}

760 Human IRE1a cDNA (NM_001433.3) was amplified from plasmid template (Addgene, \#13009) using the following oligos: 5'- agattacgctatctgtacaggcATGCCGGCCCGGCGGCTG3' and 5'- ggccgctagccogggtaccgCTTGGTTTGGGAAGCCTGGTCTCCCTGC-3' and inserted into the modified pRaichu vector (Ambrozkiewicz et al., 2020).

\section{5 pCAGIG-6XHis-eEF2}

Murine eEF2 cDNA (NM_007907.2) was amplified from cDNA library using the following oligos: 5'-gtctcatcattttggcaaagATGCATCATCATCATCATCATGTGAACTTCACAGTAGATC3' and 5'-cggccgcgatatcctcgaggCTACAGTTTGTCCAGGAAGTTG-3' and inserted into pCAGIG vector for simultaneous expression of 6 XHis-tagged eEF2 and EGFP.

\section{Nucleofection of primary neurons}

Transfection of primary cortical cells was performed using nucleofection (Lonza Bioscience) according to our previously published protocol (Ambrozkiewicz et al., 2017).

\section{Small molecule inhibitor screening}

Right after trituration, E13.5 embryonic cortical neurons prepared from Satb2 ${ }^{\mathrm{Cre} /+}$ mice, were nucleofected with pCAG-EGFP and pCAG-loxP-Stop-loxP-tdTomato plasmids and seeded at 120000 cells per well of 96-well plate. Two hours post-plating, cultures were treated with compounds at two concentrations, in technical duplicates. Cells were then cultivated until

780 DIV2, when the proportion of Satb2 ${ }^{\text {tdTom }}$ neurons normalized to EGFP positive cells was determined using FACS as described (Ambrozkiewicz et al., 2017). 


\section{Ex Utero Electroporation (EUE)}

Embryonic brains were injected with a glass capillary to fill the entire volume of a single lateral 785 embryonic ventricle with DNA (final concentration of $500 \mathrm{ng} / \mu \mathrm{L}$ ), followed by electroporation using 6 pulses of $35 \mathrm{~V}$ applied using platinum electrodes. The isolated heads were then placed in ice cold HBSS until proceeding with primary cultures.

\section{Immunocytochemistry}

790 Cells cultured on coverslips were fixed with cold $\left(4^{\circ} \mathrm{C}\right) 4 \%$ PFA, $4 \%$ sucrose (Merck) in PBS for 20 minutes in room temperature, washed three times with PBS and incubated with Blocking buffer for 30 minutes. Next, cells were incubated with primary antibodies diluted with blocking buffer accordingly for $16-20$ hours at $4^{\circ} \mathrm{C}$ with moderate shaking, followed by washing 3 times with PBS. Further, secondary antibodies coupled to appropriate fluorophore

795 diluted in blocking buffer were applied for 2 hours at room temperature. After washing 3 times with PBS, coverslips were briefly rinsed with $\mathrm{ddH}_{2} \mathrm{O}$ and mounted on Superfrost Plus glass slides (Thermo Scientific) with Immu-Mount mounting medium (Shandon, Thermo-Scientific).

Blocking buffer: 5\% horse serum (Gibco, Life Technologies), 0.3\% Triton X-100 (Roche), in 800 PBS buffer

\section{Fluorescent Noncanonical Amino Acid Tagging (FUNCAT)}

For the fluorescent labeling of newly synthesized proteins, we modified previously published methods (Tom Dieck et al., 2012). In brief, neuronal cultures were fed with 1mM HPG-

805 supplemented Methionine-free Neurobasal Medium, at DIV1 and DIV5, for the time indicated on the figures and in the figure legends. Cells were then extensively washed in PBS prior to fixation with 4\%PFA/4\% sucrose for $20 \mathrm{~min}$. Control and HPG fed neurons were then fluorescently labelled with $1 \mu \mathrm{M}$ Sulfo-Cyanine5 azide (Lumiprobe). To facilitate azide-alkyne binding we applied sCy5az in a PBS based "click solution" containing $0.2 \mathrm{mM}$ Tris(3-

810 hydroxypropyltriazolylmethyl) amine (THPTA), $20 \mathrm{mM}$ sodium L-ascorbate, and $0.2 \mathrm{mM}$ copper (II) sulfate pentahydrate (all from Sigma-Aldrich). Cells were incubated at room temperature for 20 minutes, before PBS washing and immunocytochemistry (described above).

815 Met-free Neurobasal Medium: 500 mL Met-, Lys-, Arg-free Neurobasal (Gibco, Life Technologies), $10 \mathrm{~mL}$ B-27 (Gibco, Life Technologies), $5 \mathrm{~mL}$ GlutaMAX (Gibco, Life Technologies), supplemented with final $1 \mathrm{mM} \mathrm{L-HPG} \mathrm{(Jena),} 0.8 \mathrm{mM}$ Lys, and $0.4 \mathrm{mM}$ Arg.

\section{Isolation of RNA, cDNA library preparation and RNA sequencing}

820 Tools and work surfaces used for RNA work were thoroughly cleaned with $70 \%$ ethanol (Sigma) and rinsed with RNA-Zap (Thermo Fisher) before the procedure. Embryonic brains at E18.5 were dissected in DEPC-PBS and flash-frozen in liquid nitrogen until the purification procedure. RNA was isolated using RNeasy columns (RNeasy Mini Kit, Qiagen). Further steps were performed according to the manufacturer's protocols. RNA was eluted from the

825 silica membranes using $30 \mu \mathrm{L}$ of molecular biology-grade (MB)- $\mathrm{H}_{2} \mathrm{O}$. Quality and concentration of the prepared RNA was determined using an Agilent Bioanalyzer. Single or 
paired end TruSeq Stranded total RNA libraries were made with Ribo-Zero Gold rRNA depletion and sequenced using Illumina Nextseq 500/550. Raw reads were aligned to GRCm38.p5 using STAR 2.5.3a (Dobin et al., 2013) with parameters -830 outFilterMultimapNmax 100 --winAnchormultimapNmax 100 --outSamstrandField intronMotif and the gencode vM16 basic annotation transcriptome. A single count table was generated from the resulting bam files using TETranscripts (Jin et al., 2015). Alignment pipeline was executed using Snakemake (https://snakemake.github.io/). Counts were then fit to a quasilikelihood negative binomial generalized log-linear model and tested for differential

835 expression using EdgeR (Robinson et al., 2010).

\section{Biochemical experiments}

Details of the protocols used for Preparation of cortical lysates, Protein concentration measurements, Sodium dodecyl sulfate polyacrylamide gel electrophoresis (SDS-PAGE),

840 Western blotting, Quantification of protein levels are described in our previous works (Ambrozkiewicz et al., 2018, 2020).

\section{Polysome purification}

Ribosome fractionation and polysome purification from E18.5 cortex was performed as 845 published protocol (Kraushar et al., 2021). Seven brains (14 neocortex hemispheres) of each genotype were pooled per biological replicate, with analysis in two replicates per condition.

\section{Cryosectioning}

For all histological procedures, brain sections were prepared on Leica CM3050S cryostat.

850 Prior to cryosectioning, brains were passed through a sucrose gradient to cryoprotect the tissue. Next, brains were frozen in -38 to $-40^{\circ} \mathrm{C}$ isopentane (Roth). Coronal cryosections of $50 \mu \mathrm{m}$ thickness were collected in PBS $/ 0.01 \%$ sodium azide solution. For in situ hybridization $16 \mu \mathrm{m}$ thickness sections were collected.

\section{Fluorescence in situ hybridization (FISH)}

We used RNAscope Technology to detect mRNA of Ire1 $\alpha$ according to the manufacturer's protocols (ACD). Prior to hybridization, embryonic brains at E12.5, E14.5 and E18.5 were collected in DEPC-PBS and incubated in 4\%PFA/PBS/DEPC for $16-20$ hours at $4^{\circ} \mathrm{C}$. Brains were then passed through a series of sucrose solutions (10\%-20\%-30\%/PBS) until they reach

860 osmotic equilibrium, embedded in O. C. T. Compound (Tissue-Tek) in a plastic cryoblock mold and frozen on dry ice. Coronal sections of $16 \mu \mathrm{m}$ thickness were collected using the cryostat.

\section{Immunohistochemistry}

865 Fixed brain sections were washed with PBS three times at room temperature prior to the procedure to remove the sucrose and freezing compound residue. The sections were then incubated with Blocking solution for one hour at room temperature, then with the primary antibody and DAPI diluted in blocking buffer for $16-20$ hours at $4^{\circ} \mathrm{C}$, washed in PBS three times for 30 minutes and incubated with secondary antibody diluted in the blocking buffer for

870 up to four hours at room temperature. Next, sections were incubated with PBS for 30 minutes 
three times and mounted with cover glass (Menzel-Gläser) and Immu-Mount mounting medium (Shandon, Thermo-Scientific).

875

Blocking solution: $5 \%$ horse serum, $0.5 \%(v / v)$ Triton X-100, PBS

\section{BrdU Injection and Immunohistochemistry}

Pregnant females at appropriate stages were intraperitoneally injected with BrdU, as described (Tarabykin et al., 2001). Brains were then isolated at p2 from mice of both genotypes (Control: Ire $1 \alpha^{\mathrm{flf}}$ and cKO: Ire $1 \alpha^{\mathrm{fff}} ; \mathrm{Em} \times 1^{\mathrm{Cre} / \mathrm{+}}$ ), fixed in 4\% PFA, passed through

880 sucrose gradient and frozen in isopentane bath. Free floating cryosections of $50 \mu \mathrm{m}$ thickness were placed on the SuperFrost Plus glass slides and let dry overnight at $+4^{\circ} \mathrm{C}$. Antigen retrieval was performed in boiling citrate-based Antigen Unmasking Solution (Vector, $\mathrm{pH}$ 6.0) for seven minutes in a microwave followed by 20 min of cooling on ice. Next, sections on slides were immunolabeled according to the protocol.

885

\section{Expansion Microscopy}

For the purpose of expansion of coronal brain sections to visualize morphology of EGFPexpressing neurons, we followed previously published protocol (Asano et al., 2018). For immunohistochemistry, we used anti-EGFP antibody (Rockland) at 1:200 and the secondary

890 Alexa488-coupled one at 1:100. After PBS washes, the sections were incubated with AcX crosslinker $(10 \mathrm{mg} / \mathrm{mL})$ in $150 \mathrm{mM} \mathrm{NaHCO}_{3}$ for 20 hours at room temperature, washed with PBS, incubated for one hour with monomer solution (19\% sodium acrylate, $10 \%$ acrylamide, $0.1 \%$ bis-N',N'-methylene-bisacrylamide, $0.01 \%$ 4-hydroxy-TEMPO in PBS) and gel was polymerized by addition of $0.2 \%$ APS $/ 0.2 \%$ TEMED for two hours at $37^{\circ} \mathrm{C}$. The section

895 embedded in the gel was then incubated with a proteinase $\mathrm{K}$ in a digestion buffer $(50 \mathrm{mM}$ Tris- $\mathrm{Cl}, 800 \mathrm{mM}$ guanidine- $\mathrm{Cl}, 2 \mathrm{mM} \mathrm{CaCl}_{2}, 0.5 \%$ Triton $\mathrm{X}-100$ in $\mathrm{pH} 8.0$ ) for 20 hours at $37^{\circ} \mathrm{C}$ with gentle agitation. Next, section was transferred to a Petri dish with $\mathrm{dd}_{2} \mathrm{O}$, which was changed at least five times for two days. The expansion factor was determined before mounting of the specimen. Sections were then transferred onto the glass-bottom chambers

900 (Ibidi) and imaged using Leics Sp8 confocal.

\section{In utero electroporation (IUE)}

Prior to IUE, DNA was diluted in TE buffer and mixed with $0.1 \%$ Fast Green FCF (SigmaAldrich). Final concentration of DNA used for transfecting cortical progenitors was 200-500 $905 \mathrm{ng} / \mu \mathrm{L}$. The details on the procedure are exactly as we described before (Ambrozkiewicz et al., 2018).

\section{Antibodies}

For Western blotting, all antibodies were diluted 1:750, apart from anti- $\beta$-actin, which was 910 used at 1:2000, in 5\% non-fat dry milk in TBS-T buffer (TBS buffer with 0.1\% Tween-20). Antibodies against phospho-proteins and anti-Ire1 $\alpha$ were diluted in 3\%BSA in TBS-T buffer. Secondary antibodies coupled with HRP were diluted 1:10000 in the same buffer as the primary ones. 
For immunocytochemistry, all primary antibodies were diluted 1:300 in the blocking buffer,

915 and the appropriate fluorophore-conjugated secondary antibodies were used at 1:750.

For immunohistochemistry, primary antibodies and the respective fluorophore-linked secondary ones were diluted 1:500 in the blocking buffer.

\section{Data analysis}

920 Details concerning Quantitative Western blotting, Axon counting assays in vitro, Neuronal morphometry, Quantification of distribution of cortical neurons and polarity classification are described in detailed in our previous publications (Ambrozkiewicz et al., 2018, 2020).

\section{Confocal imaging of immunostaining signals and analysis}

925 Images of primary cell cultures and brain sections after immunostaining were acquired using confocal Leica SL, Leica Sp8 or Zeiss Spinning Disc Microscope.

\section{Colocalization with identity markers}

Neuronal identity on brain sections was quantified as fraction of all EGFP-expressing

930 neurons. Identity of primary cells on coverslips was determined as fraction of fluorescent reporter-expressing cell. Based on the numbers of dsRed- or EGFP-expressing cells, we either quantified at least 4 fields of view for high-density cultures, or imaged a given number of cells (indicated on the bar graphs or Table S1) and determined the number of cells expressing a marker protein for cultures with sparse labeling, i.e. after EUE. Compare Fig.

935 S6.

\section{Laminar positioning (\% of CP)}

We based our positioning analysis on previous published reports (Oberst et al., 2019). To determine the position of neurons in the cortical plate, confocal images of EGFP signals were

940 first transformed so that the pia is perpendicularly oriented to the horizontal axis. Next, positions of neurons were marked in Fiji using the Cell Counter plug-in. Using the ycoordinate, we then expressed the position of a given cell relative to the size of the CP and plotted it as $\% \mathrm{CP}$, with $0 \%$ being the bottom of the cortical plate / the subplate (SP) and a $100 \%$ being the pial surface / the marginal zone (MZ). Positions of all cells were then plotted

945 individually on the graph. Only brains with comparable electroporation efficiencies were analyzed.

\section{Quantification of fluorescence intensity}

For quantifications of fluorescent intensity, we defined a region of interest based on the

950 fluorescent marker, i.e. dsRed or EGFP signals. Using Fiji software, the raw integrated density was then normalized to the cell surface area of the outlined cell compartment. For our quantifications, we imaged the primary cell cultures as Z-stacks and for the analysis, we used maximum intensity projections.

\section{Gene Set Enrichment Analysis (GSEA)}

GSEA was performed on total counts from cKO and control polysome fraction RNAseq in classic mode, testing set, the difference of classes metric, over 1000 permutations with the 
c5.go.mf.v7.2.symbols.gmt (molecular function) annotation. This gave 406/772 gene sets upregulated in cKO polysomes where 158 gene sets were significant at FDR $<25 \%$. Detailed 960 results are presented in the Table S2.

\section{Statistical analyses}

All statistics were performed using Prism Graph Pad software. Detailed information on statistics can be found in the supplementary material. Description of statistical tests, definition 965 of center, dispersion, precision and definition of significance are also listed in the figure legends. Briefly, distribution of datapoints was determined using D'Agostino and Pearson normality test. For normally distributed data, we used unpaired two-tailed t-test, or one/twoway ANOVA with Bonferroni post hoc test; for data with not normal distribution, we used Mann-Whitney test to compare two groups, or Kruskal-Wallis test with Dunn's Multiple 970 comparison test for comparison between multiple groups, unless specified otherwise. For detailed information on all numerical values, sample size and statistical tests used in all experiments, see Table S1.

KEY RESOURCES TABLE

\begin{tabular}{|c|c|c|}
\hline REAGENT or RESOURCE & SOURCE & IDENTIFIER \\
\hline \multicolumn{3}{|l|}{ Antibodies } \\
\hline Mouse anti-Tau-1 & Millipore & $\begin{array}{l}\text { Clone PC16C, } \\
\text { MAB3420, } \\
\text { RRID:AB_94855 }\end{array}$ \\
\hline Chicken anti-MAP2 & Novus & RRID:AB_350528 \\
\hline Goat anti-GFP & Rockland & $\begin{array}{l}\text { 600-101-215M, } \\
\text { RRID:AB_2612804 }\end{array}$ \\
\hline Chicken anti-GFP & Abcam & $\begin{array}{l}\text { ab13970, } \\
\text { RRID:AB_300798 }\end{array}$ \\
\hline Rabbit anti-Ki67 & Gift from Prim Singh & $\mathrm{N} / \mathrm{A}$ \\
\hline Rabbit anti-Pax6 & Millipore & $\begin{array}{l}\text { AB2237, } \\
\text { RRID:AB_1587367 }\end{array}$ \\
\hline Rabbit anti-Tbr1 & Abcam & $\begin{array}{l}\text { ab31940, } \\
\text { RRID:AB_2200219 }\end{array}$ \\
\hline Rabbit anti-Satb2 & Generated in-house & $\mathrm{N} / \mathrm{A}$ \\
\hline Mouse anti-Satb2 & SCBT & $\begin{array}{l}\text { sc-81376, } \\
\text { RRID:AB_1129287 }\end{array}$ \\
\hline Rabbit anti-Cux1 (CDP) & SCBT & $\begin{array}{l}\text { M-222; } \\
\text { RRID:AB_2261231 }\end{array}$ \\
\hline Rat anti-CTIP2 & Abcam & $\begin{array}{l}\text { 25B6; } \\
\text { RRID:AB_2064130 }\end{array}$ \\
\hline Rabbit anti-Bcl11b & CST & $\begin{array}{l}\text { D6F1, } \\
\text { RRID:AB_2797823 }\end{array}$ \\
\hline Rabbit anti-Ire1a & Abcam & $\begin{array}{l}\text { ab37073; } \\
\text { RRID:AB_775780 }\end{array}$ \\
\hline
\end{tabular}




\begin{tabular}{|c|c|c|}
\hline Rabbit anti-Ire1 $\alpha$ & CST & $\begin{array}{l}\text { 14C10, } \\
\text { RRID:AB_2055392 }\end{array}$ \\
\hline Rat anti-BrdU & Abcam & $\begin{array}{l}\text { ab6326, } \\
\text { RRID:AB_305426 }\end{array}$ \\
\hline Mouse anti-Gapdh & Hytest & $\begin{array}{l}\text { 5G4-6C5; } \\
\text { RRID:AB_1616722 }\end{array}$ \\
\hline Mouse anti-AnkyrinG & $\begin{array}{l}\text { Antibodies } \\
\text { Incorporated }\end{array}$ & $\begin{array}{l}\text { 75-146; } \\
\text { RRID:AB_10673030 }\end{array}$ \\
\hline Mouse anti-Sodium Channel Pan & Sigma-Aldrich & $\begin{array}{l}\text { S8809; } \\
\text { RRID:AB_477552 }\end{array}$ \\
\hline Rabbit anti-RFP & Abcam & $\begin{array}{l}\text { ab62341; } \\
\text { RRID:AB_945213 }\end{array}$ \\
\hline Rabbit anti-FoxP2 & Abcam & $\begin{array}{l}\text { ab16046; } \\
\text { RRID:AB_2107107 }\end{array}$ \\
\hline Rabbit anti-P-S6 Ribosomal protein & CST & $\begin{array}{l}\text { 2211S; } \\
\text { RRID:AB_331679 }\end{array}$ \\
\hline Rabbit anti-FMRP & CST & $\begin{array}{l}\text { 4317S; } \\
\text { RRID:AB_1903978 }\end{array}$ \\
\hline Rabbit anti-elF2 $\alpha$ & CST & $\begin{array}{l}\text { 9722S; } \\
\text { RRID:AB_2230924 }\end{array}$ \\
\hline Rabbit anti-elF2aPSer51 & CST & $\begin{array}{l}\text { 3398S; } \\
\text { RRID:AB_2096481 }\end{array}$ \\
\hline Rabbit anti-Xbp1S & CST & $\begin{array}{l}\text { 12782; } \\
\text { RRID:AB_2687943 }\end{array}$ \\
\hline Mouse anti-Vinculin & SCBT & $\begin{array}{l}\text { SC-73614; } \\
\text { RRID:AB_1131294 }\end{array}$ \\
\hline Mouse anti- $\beta$-actin & Millipore & $\begin{array}{l}\text { MAB1501R; } \\
\text { RRID:AB_2223041 }\end{array}$ \\
\hline Mouse anti- $\beta$-tubulin & BioLegend & $\begin{array}{l}\text { 801201; } \\
\text { RRID:AB_2313773 }\end{array}$ \\
\hline Mouse anti-eEF2PThr56 & CST & $\begin{array}{l}\text { 2331S; } \\
\text { RRID:AB_10015204 }\end{array}$ \\
\hline Mouse anti-eEF2 & CST & $\begin{array}{l}\text { 2332S; } \\
\text { RRID:AB_10693546 }\end{array}$ \\
\hline Rat anti-RFP & Chromotek & $\begin{array}{l}\text { 5f8-100; } \\
\text { RRID:AB_2336064 }\end{array}$ \\
\hline Donkey lgG anti-Mouse IgG (H+L)-HRPO & $\begin{array}{l}\text { Jackson } \\
\text { Immunoresearch }\end{array}$ & $\begin{array}{l}\text { 715-035-150; } \\
\text { RRID:AB_2340770 }\end{array}$ \\
\hline Donkey lgG anti-Rabbit lgG (H+L)-HRPO & $\begin{array}{l}\text { Jackson } \\
\text { Immunoresearch }\end{array}$ & $\begin{array}{l}\text { 711-035-152; } \\
\text { RRID:AB_10015282 }\end{array}$ \\
\hline Donkey anti-goat AlexaFluor-488 & $\begin{array}{l}\text { Jackson } \\
\text { Immunoresearch }\end{array}$ & $\begin{array}{l}\text { 705-546-147; } \\
\text { RRID:AB_2340430 }\end{array}$ \\
\hline
\end{tabular}




\begin{tabular}{|c|c|c|}
\hline Donkey anti-rat AlexaFluor-488 & $\begin{array}{l}\text { Jackson } \\
\text { Immunoresearch }\end{array}$ & $\begin{array}{l}\text { 712-545-150; } \\
\text { RRID:AB_2340683 }\end{array}$ \\
\hline Donkey anti-rabbit AlexaFluor-Cy3 & $\begin{array}{l}\text { Jackson } \\
\text { Immunoresearch }\end{array}$ & $\begin{array}{l}\text { 711-167-003; } \\
\text { RRID:AB_2340606 }\end{array}$ \\
\hline Donkey anti-rabbit AlexaFluor-Cy3 & $\begin{array}{l}\text { Jackson } \\
\text { Immunoresearch }\end{array}$ & $\begin{array}{l}\text { 711-165-152; } \\
\text { RRID:AB_2307443 }\end{array}$ \\
\hline Donkey anti-rat AlexaFluor-Cy3 & $\begin{array}{l}\text { Jackson } \\
\text { Immunoresearch }\end{array}$ & $\begin{array}{l}\text { 712-165-153; } \\
\text { RRID:AB_2340667 }\end{array}$ \\
\hline Donkey anti-rat AlexaFluor-647 & $\begin{array}{l}\text { Jackson } \\
\text { Immunoresearch }\end{array}$ & $\begin{array}{l}\text { 712-175-153; } \\
\text { RRID:AB_2340672 }\end{array}$ \\
\hline Donkey anti-mouse AlexaFluor-Cy3 & $\begin{array}{l}\text { Jackson } \\
\text { Immunoresearch }\end{array}$ & $\begin{array}{l}\text { 715-165-150; } \\
\text { RRID:AB_2340813 }\end{array}$ \\
\hline Donkey anti-rabbit AlexaFluor-647 & $\begin{array}{l}\text { Jackson } \\
\text { Immunoresearch }\end{array}$ & $\begin{array}{l}\text { 711-605-152; } \\
\text { RRID:AB_2492288 }\end{array}$ \\
\hline Donkey anti-chicken AlexaFlour-647 & $\begin{array}{l}\text { Jackson } \\
\text { Immunoresearch }\end{array}$ & $\begin{array}{l}\text { 703-605-155; } \\
\text { RRID:AB_2340379 }\end{array}$ \\
\hline Draq5 & Biostatus Limited & RRID:AB_2314341 \\
\hline \multicolumn{3}{|l|}{ Bacterial and Virus Strains } \\
\hline Escherichia coli TOP10 competent cells & Invitrogen & C404052 \\
\hline pLKO-EGFP Lentivirus & $\begin{array}{l}\text { Charité Viral Core } \\
\text { Facility }\end{array}$ & $\mathrm{N} / \mathrm{A}$ \\
\hline pLKO-Cre Lentivirus & $\begin{array}{l}\text { Charité Viral Core } \\
\text { Facility }\end{array}$ & $\mathrm{N} / \mathrm{A}$ \\
\hline \multicolumn{3}{|l|}{ FISH Probes } \\
\hline RNAscope Probe Mm-Ern1 & ACD, Bio-techne & 438031 \\
\hline \multicolumn{3}{|l|}{ Biological Samples } \\
\hline \multicolumn{3}{|c|}{ Chemicals, Peptides, and Recombinant Proteins } \\
\hline Cycloheximide (CHX) & Merck & 01810 \\
\hline Dimethylsufoxide (DMSO) & Merck & D8418 \\
\hline L-Homopropargylglycine (L-HPG) & Jena Bioscience & CLK-1067 \\
\hline FlashTag-488 & ThermoFisher & C34554 \\
\hline \multicolumn{3}{|l|}{ Critical Commercial Assays } \\
\hline $\begin{array}{l}\text { RNAscope Multiplex Fluorescent Reagent } \\
\text { Kit v2 }\end{array}$ & ACD, Bio-techne & 323100 \\
\hline NEBuilder HF DNA Assembly Cloning Kit & $\begin{array}{l}\text { New } \\
\text { BioLabs }\end{array}$ & E5520 \\
\hline $\begin{array}{l}\text { Translation Initiation Complex Antibody } \\
\text { Sampler Kit }\end{array}$ & CST & 4763 \\
\hline \multicolumn{3}{|l|}{ Deposited Data } \\
\hline \multicolumn{3}{|l|}{ Experimental Models: Cell Lines } \\
\hline
\end{tabular}




\begin{tabular}{|c|c|c|}
\hline HEK293T & $\begin{array}{l}\text { Leibniz Institute } \\
\text { DSMZ-German } \\
\text { Collection of } \\
\text { Microorganisms and } \\
\text { Cell Cultures }\end{array}$ & $\begin{array}{l}\text { ACC 635; RRID: } \\
\text { CVCL_0063 }\end{array}$ \\
\hline Primary murine cortical cells & This paper & N/A \\
\hline \multicolumn{3}{|l|}{ Experimental Models: Organisms/Strains } \\
\hline Ire $1 a^{f / f}$ mouse line & $\begin{array}{l}\text { (Iwawaki et al., } \\
2009)\end{array}$ & N/A \\
\hline Ire $1 a^{\text {fff }} \mathrm{Em} \times 1^{\mathrm{Cre} / \mathrm{t}}$ mouse line & This paper & N/A \\
\hline Satb2 ${ }^{\text {Cre/+ }}$ mouse line & $\begin{array}{l}\text { (Britanova et al., } \\
2006)\end{array}$ & RRID:MGI:3695609 \\
\hline NMRI wild type mouse strain & $\begin{array}{l}\text { Charles Rivers and } \\
\text { Janvier Labs }\end{array}$ & $\begin{array}{l}\text { RRID:IMSR_TAC:nm } \\
\text { ri }\end{array}$ \\
\hline \multicolumn{3}{|l|}{ Recombinant DNA } \\
\hline Mouse cortex cDNA library (e12, e14, e16) & This paper & $\mathrm{N} / \mathrm{A}$ \\
\hline pCX::myr-Venus & $\begin{array}{ll}\text { gift from } & \text { A. } \\
\text { Hadjantonakis } & \end{array}$ & N/A \\
\hline pCAG & $\begin{array}{l}\text { gift from Jun-ichi } \\
\text { Miyazaki }\end{array}$ & N/A \\
\hline pCAGIG & & Addgene \\
\hline pRaichu205X & $\begin{array}{l}\text { gift from Michiyuki } \\
\text { Matsuda }\end{array}$ & N/A \\
\hline pCAG-EGFP & (Lois et al., 2002) & Addgene \#14883 \\
\hline pCAG-flox-Stop-flox-TdTomato & gift from B. Eickholt & N/A \\
\hline pCAG-flox-Stop-flox-EGFP & gift from B. Eickholt & N/A \\
\hline pCAG-EGFPiCre & gift from R. Huganir & N/A \\
\hline pCAG-Cre & $\begin{array}{ll}\text { gift from } & \text { S. } \\
\text { Parathasarathy } & \end{array}$ & N/A \\
\hline pcDNA3.EGFP-IRE1 $\alpha$ & (Lipson et al., 2006) & Addgene $\# 13009$ \\
\hline pRai-HA-Ire1 $\alpha$ & This paper & $\mathrm{N} / \mathrm{A}$ \\
\hline pNeuroD-GFP & gift from G. Fishell & N/A \\
\hline pNeuroD-Cre & $\begin{array}{ll}\text { gift from } & \mathrm{S} . \\
\text { Parathasarathy } & \end{array}$ & \\
\hline pX335-sgRNA-EF(Cas9n)-Eif4a1-R & (Ochiai et al., 2020) & Addgene \#122345 \\
\hline pX335-sgRNA-EF(Cas9n)-Eif4a1-L & (Ochiai et al., 2020) & Addgene \#122346 \\
\hline pCAGIG-6XHis-eEF2 & This paper & N/A \\
\hline EGFP-Sec61 $\beta$ & $\begin{array}{l}\text { gift from Tom } \\
\text { Rapoport }\end{array}$ & Addgene \#15108 \\
\hline pCAG-Intron-dsRed & gift from $\mathrm{H}$. Fuchs & N/A \\
\hline \multicolumn{3}{|l|}{ Software and Algorithms } \\
\hline Fiji & imagej.net & N/A \\
\hline GraphPad Prism & GraphPad Software & N/A \\
\hline
\end{tabular}




\begin{tabular}{|l|l|l|}
\hline Photoshop & Adobe & N/A \\
\hline Snakemake & $\begin{array}{l}\text { https://snakemake.g } \\
\text { ithub.io/ }\end{array}$ & N/A \\
\hline STAR & $\begin{array}{l}\text { https://github.com/al } \\
\text { exdobin/STAR }\end{array}$ & N/A \\
\hline TETranscripts & $\begin{array}{l}\text { https://github.com/m } \\
\text { hammell- } \\
\text { laboratory/TEtranscr } \\
\text { ipts }\end{array}$ & N/A \\
\hline EdgeR & $\begin{array}{l}\text { https://bioconductor. } \\
\text { org/packages/releas } \\
\text { e/bioc/html/edgeR.h } \\
\text { tml }\end{array}$ & N/A \\
\hline GSEA & $\begin{array}{l}\text { https://www.gsea- } \\
\text { msigdb.org/gsea/ind } \\
\text { ex.jsp }\end{array}$ & N/A \\
\hline
\end{tabular}

975 N/A not available

\section{SUPPLEMENTARY TABLES}

Table S1. Numerical values, sample size and statistics for experiments in this paper.

Table S2. GSEA report for RNA upregulated in cKO polysomes. 980

\section{REFERENCES}

Acosta-Alvear, D., Karagöz, G.E., Fröhlich, F., Li, H., Walther, T.C., and Walter, P. (2018). The unfolded protein response and endoplasmic reticulum protein targeting machineries converge on the stress sensor IRE1. Elife 7.

985 Alcamo, E.A., Chirivella, L., Dautzenberg, M., Dobreva, G., Fariñas, I., Grosschedl, R., and McConnell, S.K. (2008). Satb2 Regulates Callosal Projection Neuron Identity in the Developing Cerebral Cortex. Neuron 57, 364-377.

Ambrozkiewicz, M.C., Bessa, P., Salazar-Lázaro, A., Salina, V., and Tarabykin, V. (2017). Satb2(Cre/+) mouse as a tool to investigate cell fate determination in the developing 990 neocortex. J. Neurosci. Methods 291, 113-121.

Ambrozkiewicz, M.C., Schwark, M., Kishimoto-Suga, M., Borisova, E., Hori, K., SalazarLázaro, A., Rusanova, A., Altas, B., Piepkorn, L., Bessa, P., et al. (2018). Polarity Acquisition in Cortical Neurons Is Driven by Synergistic Action of Sox9-Regulated Wwp1 and Wwp2 E3 Ubiquitin Ligases and Intronic miR-140. Neuron 100, 1097-1115.e15.

995 Ambrozkiewicz, M.C., Borisova, E., Schwark, M., Ripamonti, S., Schaub, T., Smorodchenko, A., Weber, A.I., Rhee, H.J., Altas, B., Yilmaz, R., et al. (2020). The murine ortholog of Kaufman oculocerebrofacial syndrome protein Ube $3 b$ regulates synapse number by ubiquitinating Ppp3cc. Molecular Psychiatry.

Andersen, C.B.F. (2006). Structure of the Exon Junction Core Complex with a Trapped 1000 DEAD-Box ATPase Bound to RNA. Science 313, 1968-1972. 
Arimura, N., and Kaibuchi, K. (2005). Key regulators in neuronal polarity. Neuron 48, 881884.

Arlotta, P., Molyneaux, B.J., Chen, J., Inoue, J., Kominami, R., and Macklis, J.D. (2005). Neuronal subtype-specific genes that control corticospinal motor neuron development in vivo. 1005 Neuron 45, 207-221.

Asano, S.M., Gao, R., Wassie, A.T., Tillberg, P.W., Chen, F., and Boyden, E.S. (2018). Expansion Microscopy: Protocols for Imaging Proteins and RNA in Cells and Tissues. Current Protocols in Cell Biology 80.

Barnes, A.P., and Polleux, F. (2009). Establishment of axon-dendrite polarity in developing 1010 neurons. Annu. Rev. Neurosci. 32, 347-381.

Barnes, A.P., Lilley, B.N., Pan, Y.A., Plummer, L.J., Powell, A.W., Raines, A.N., Sanes, J.R., and Polleux, F. (2007). LKB1 and SAD kinases define a pathway required for the polarization of cortical neurons. Cell 129, 549-563.

Beatty, K.E., and Tirrell, D.A. (2008). Two-color labeling of temporally defined protein 1015 populations in mammalian cells. Bioorg Med Chem Lett 18, 5995-5999.

Biever, A., Glock, C., Tushev, G., Ciirdaeva, E., Dalmay, T., Langer, J.D., and Schuman, E.M. (2020). Monosomes actively translate synaptic mRNAs in neuronal processes. Science 367 , eaay4991.

Britanova, O., Depew, M.J., Schwark, M., Thomas, B.L., Miletich, I., Sharpe, P., and 1020 Tarabykin, V. (2006). Satb2 Haploinsufficiency Phenocopies 2q32-q33 Deletions, whereas Loss Suggests a Fundamental Role in the Coordination of Jaw Development. The American Journal of Human Genetics 79, 668-678.

Britanova, O., de Juan Romero, C., Cheung, A., Kwan, K.Y., Schwark, M., Gyorgy, A., Vogel, T., Akopov, S., Mitkovski, M., Agoston, D., et al. (2008). Satb2 is a postmitotic determinant 1025 for upper-layer neuron specification in the neocortex. Neuron 57, 378-392.

Carter, S.D., Tran, N.-H., De Mazière, A., Ashkenazi, A., Klumperman, J., Jensen, G.J., and Walter, P. (2021). The stress-sensing domain of activated IRE1 $\alpha$ forms helical filaments in narrow ER membrane tubes (Cell Biology).

Chen, B., Schaevitz, L.R., and McConnell, S.K. (2005). Fezl regulates the differentiation and 1030 axon targeting of layer 5 subcortical projection neurons in cerebral cortex. Proc Natl Acad Sci U S A 102, 17184-17189.

Cox, J.S., Shamu, C.E., and Walter, P. (1993). Transcriptional induction of genes encoding endoplasmic reticulum resident proteins requires a transmembrane protein kinase. Cell 73, 1197-1206.

1035 Di Prisco, G.V., Huang, W., Buffington, S.A., Hsu, C.-C., Bonnen, P.E., Placzek, A.N., Sidrauski, C., Krnjević, K., Kaufman, R.J., Walter, P., et al. (2014). Translational control of mGluR-dependent long-term depression and object-place learning by elF2 $\alpha$. Nat Neurosci 17, 1073-1082. 
Dieterich, D.C., Hodas, J.J.L., Gouzer, G., Shadrin, I.Y., Ngo, J.T., Triller, A., Tirrell, D.A., 1040 and Schuman, E.M. (2010). In situ visualization and dynamics of newly synthesized proteins in rat hippocampal neurons. Nat Neurosci 13, 897-905.

Dobin, A., Davis, C.A., Schlesinger, F., Drenkow, J., Zaleski, C., Jha, S., Batut, P., Chaisson, M., and Gingeras, T.R. (2013). STAR: ultrafast universal RNA-seq aligner. Bioinformatics 29, 15-21.

1045 Doultsinos, D., McMahon, M., Voutetakis, K., Obacz, J., Pineau, R., Le Reste, P.-J., Obiedat, A., Samal, J., Patterson, J.B., Zheng, Q., et al. (2019). Constitutive UPR ER activation sustains tumor cell differentiation (Cancer Biology).

Eshraghi, M., Karunadharma, P.P., Blin, J., Shahani, N., Ricci, E.P., Michel, A., Urban, N.T., Galli, N., Sharma, M., Ramírez-Jarquín, U.N., et al. (2021). Mutant Huntingtin stalls 1050 ribosomes and represses protein synthesis in a cellular model of Huntington disease. Nat Commun 12, 1461.

Farías, G.G., Fréal, A., Tortosa, E., Stucchi, R., Pan, X., Portegies, S., Will, L., Altelaar, M., and Hoogenraad, C.C. (2019). Feedback-Driven Mechanisms between Microtubules and the Endoplasmic Reticulum Instruct Neuronal Polarity. Neuron 102, 184-201.e8.

1055 Giusti, S.A., Vogl, A.M., Brockmann, M.M., Vercelli, C.A., Rein, M.L., Trümbach, D., Wurst, W., Cazalla, D., Stein, V., Deussing, J.M., et al. (2014). MicroRNA-9 controls dendritic development by targeting REST. ELife 3.

Götz, M., Stoykova, A., and Gruss, P. (1998). Pax6 controls radial glia differentiation in the cerebral cortex. Neuron 21, 1031-1044.

1060 Grinshtein, N., Rioseco, C.C., Marcellus, R., Uehling, D., Aman, A., Lun, X., Muto, O., Podmore, L., Lever, J., Shen, Y., et al. (2016). Small molecule epigenetic screen identifies novel EZH2 and HDAC inhibitors that target glioblastoma brain tumor-initiating cells. Oncotarget 7, 59360-59376.

Hafner, A.-S., Donlin-Asp, P.G., Leitch, B., Herzog, E., and Schuman, E.M. (2019). Local 1065 protein synthesis is a ubiquitous feature of neuronal pre- and postsynaptic compartments. Science 364, eaau3644.

Hinnebusch, A.G., Ivanov, I.P., and Sonenberg, N. (2016). Translational control by 5'untranslated regions of eukaryotic mRNAs. Science 352, 1413-1416.

Iwawaki, T., Akai, R., Yamanaka, S., and Kohno, K. (2009). Function of IRE1 alpha in the 1070 placenta is essential for placental development and embryonic viability. Proc Natl Acad Sci U S A 106, 16657-16662.

Jin, Y., Tam, O.H., Paniagua, E., and Hammell, M. (2015). TEtranscripts: a package for including transposable elements in differential expression analysis of RNA-seq datasets. Bioinformatics 31, 3593-3599.

1075 Kimmel, J.C., Penland, L., Rubinstein, N.D., Hendrickson, D.G., Kelley, D.R., and Rosenthal, A.Z. (2019). Murine single-cell RNA-seq reveals cell-identity- and tissue-specific trajectories of aging. Genome Res. 29, 2088-2103. 
Kraushar, M.L., Thompson, K., Wijeratne, H.R.S., Viljetic, B., Sakers, K., Marson, J.W., Kontoyiannis, D.L., Buyske, S., Hart, R.P., and Rasin, M.-R. (2014). Temporally defined 1080 neocortical translation and polysome assembly are determined by the RNA-binding protein $\mathrm{Hu}$ antigen R. Proceedings of the National Academy of Sciences 111, E3815-E3824.

Kraushar, M.L., Krupp, F., Harnett, D., Turko, P., Ambrozkiewicz, M.C., Sprink, T., Imami, K., Günnigmann, M., Zinnall, U., Vieira-Vieira, C.H., et al. (2021). Protein Synthesis in the Developing Neocortex at Near-Atomic Resolution Reveals Ebp1-Mediated Neuronal 1085 Proteostasis at the 60S Tunnel Exit. Molecular Cell 81, 304-322.e16.

Krishnamoorthy, T., Pavitt, G.D., Zhang, F., Dever, T.E., and Hinnebusch, A.G. (2001). Tight Binding of the Phosphorylated a Subunit of Initiation Factor 2 (elF2a) to the Regulatory Subunits of Guanine Nucleotide Exchange Factor elF2B Is Required for Inhibition of Translation Initiation. Mol. Cell. Biol. 21, 5018-5030.

1090 La Manno, G., Siletti, K., Furlan, A., Gyllborg, D., Vinsland, E., Langseth, C.M., Khven, I., Johnsson, A., Nilsson, M., Lönnerberg, P., et al. (2020). Molecular architecture of the developing mouse brain (Developmental Biology).

Lee, K.P.K., Dey, M., Neculai, D., Cao, C., Dever, T.E., and Sicheri, F. (2008). Structure of the dual enzyme Ire1 reveals the basis for catalysis and regulation in nonconventional RNA 1095 splicing. Cell 132, 89-100.

Lipson, K.L., Fonseca, S.G., Ishigaki, S., Nguyen, L.X., Foss, E., Bortell, R., Rossini, A.A., and Urano, F. (2006). Regulation of insulin biosynthesis in pancreatic beta cells by an endoplasmic reticulum-resident protein kinase IRE1. Cell Metabolism 4, 245-254.

Lois, C., Hong, E.J., Pease, S., Brown, E.J., and Baltimore, D. (2002). Germline transmission 1100 and tissue-specific expression of transgenes delivered by lentiviral vectors. Science 295, 868-872.

Luo, L., Ambrozkiewicz, M.C., Benseler, F., Chen, C., Dumontier, E., Falkner, S., Furlanis, E., Gomez, A.M., Hoshina, N., Huang, W.-H., et al. (2020). Optimizing Nervous SystemSpecific Gene Targeting with Cre Driver Lines: Prevalence of Germline Recombination and 1105 Influencing Factors. Neuron 106, 37-65.e5.

Malatesta, P., Hartfuss, E., and Götz, M. (2000). Isolation of radial glial cells by fluorescentactivated cell sorting reveals a neuronal lineage. Development 127, 5253-5263.

McKenna, W.L., Ortiz-Londono, C.F., Mathew, T.K., Hoang, K., Katzman, S., and Chen, B. (2015). Mutual regulation between Satb2 and Fezf2 promotes subcerebral projection neuron 1110 identity in the developing cerebral cortex. Proc Natl Acad Sci U S A 112, 11702-11707.

Molyneaux, B.J., Arlotta, P., Hirata, T., Hibi, M., and Macklis, J.D. (2005). Fezl is required for the birth and specification of corticospinal motor neurons. Neuron 47, 817-831.

Mori, K., Ma, W., Gething, M.J., and Sambrook, J. (1993). A transmembrane protein with a $\mathrm{cdc} 2+/ \mathrm{CDC} 28-$ related kinase activity is required for signaling from the ER to the nucleus. Cell $111574,743-756$.

Nowakowski, T.J., Bhaduri, A., Pollen, A.A., Alvarado, B., Mostajo-Radji, M.A., Di Lullo, E., Haeussler, M., Sandoval-Espinosa, C., Liu, S.J., Velmeshev, D., et al. (2017). Spatiotemporal 
gene expression trajectories reveal developmental hierarchies of the human cortex. Science 358, 1318-1323.

1120 Oberst, P., Fièvre, S., Baumann, N., Concetti, C., Bartolini, G., and Jabaudon, D. (2019). Temporal plasticity of apical progenitors in the developing mouse neocortex. Nature 573, 370-374.

Ochiai, H., Hayashi, T., Umeda, M., Yoshimura, M., Harada, A., Shimizu, Y., Nakano, K., Saitoh, N., Liu, Z., Yamamoto, T., et al. (2020). Genome-wide kinetic properties of 1125 transcriptional bursting in mouse embryonic stem cells. Science Advances 6, eaaz6699.

Rakic, P. (1972). Mode of cell migration to the superficial layers of fetal monkey neocortex. J Comp Neurol 145, 61-83.

Robinson, M.D., McCarthy, D.J., and Smyth, G.K. (2010). edgeR: a Bioconductor package for differential expression analysis of digital gene expression data. Bioinformatics 26, 1391130140.

Schneider-Poetsch, T., Ju, J., Eyler, D.E., Dang, Y., Bhat, S., Merrick, W.C., Green, R., Shen, B., and Liu, J.O. (2010). Inhibition of eukaryotic translation elongation by cycloheximide and lactimidomycin. Nat Chem Biol 6, 209-217.

Scholzen, T., Endl, E., Wohlenberg, C., van der Sar, S., Cowell, I.G., Gerdes, J., and Singh, 1135 P.B. (2002). The Ki-67 protein interacts with members of the heterochromatin protein 1 (HP1) family: a potential role in the regulation of higher-order chromatin structure. J. Pathol. 196, 135-144.

Sheen, V.L. (2001). Mutations in the X-linked filamin 1 gene cause periventricular nodular heterotopia in males as well as in females. Human Molecular Genetics 10, 1775-1783.

1140 Shinmyo, Y., Tanaka, S., Tsunoda, S., Hosomichi, K., Tajima, A., and Kawasaki, H. (2016). CRISPR/Cas9-mediated gene knockout in the mouse brain using in utero electroporation. Scientific Reports 6.

Starck, S.R., Tsai, J.C., Chen, K., Shodiya, M., Wang, L., Yahiro, K., Martins-Green, M., Shastri, N., and Walter, P. (2016). Translation from the 5' untranslated region shapes the 1145 integrated stress response. Science 351, aad3867-aad3867.

Tarabykin, V., Stoykova, A., Usman, N., and Gruss, P. (2001). Cortical upper layer neurons derive from the subventricular zone as indicated by Svet1 gene expression. Development 128, 1983-1993.

Telley, L., Agirman, G., Prados, J., Amberg, N., Fièvre, S., Oberst, P., Bartolini, G., Vitali, I., 1150 Cadilhac, C., Hippenmeyer, S., et al. (2019). Temporal patterning of apical progenitors and their daughter neurons in the developing neocortex. Science 364.

Tirasophon, W., Welihinda, A.A., and Kaufman, R.J. (1998). A stress response pathway from the endoplasmic reticulum to the nucleus requires a novel bifunctional protein kinase/endoribonuclease (Ire1p) in mammalian cells. Genes Dev 12, 1812-1824. 
1155 Tom Dieck, S., Müller, A., Nehring, A., Hinz, F.I., Bartnik, I., Schuman, E.M., and Dieterich, D.C. (2012). Metabolic labeling with noncanonical amino acids and visualization by chemoselective fluorescent tagging. Curr Protoc Cell Biol Chapter 7, Unit7.11.

Urra, H., Henriquez, D.R., Cánovas, J., Villarroel-Campos, D., Carreras-Sureda, A., Pulgar, E., Molina, E., Hazari, Y.M., Limia, C.M., Alvarez-Rojas, S., et al. (2018). IRE1a governs 1160 cytoskeleton remodelling and cell migration through a direct interaction with filamin A. Nat. Cell Biol. 20, 942-953.

Vattem, K.M., and Wek, R.C. (2004). Reinitiation involving upstream ORFs regulates ATF4 mRNA translation in mammalian cells. Proceedings of the National Academy of Sciences 101, 11269-11274.

1165 Yoshida, H., Matsui, T., Yamamoto, A., Okada, T., and Mori, K. (2001). XBP1 mRNA Is Induced by ATF6 and Spliced by IRE1 in Response to ER Stress to Produce a Highly Active Transcription Factor. Cell 107, 881-891.

Zahr, S.K., Yang, G., Kazan, H., Borrett, M.J., Yuzwa, S.A., Voronova, A., Kaplan, D.R., and Miller, F.D. (2018). A Translational Repression Complex in Developing Mammalian Neural 1170 Stem Cells that Regulates Neuronal Specification. Neuron 97, 520-537.e6. 